\title{
Global Behavior for a Strongly Coupled Predator-Prey Model with One Resource and Two Consumers
}

\author{
Yujuan Jiao, ${ }^{1,2}$ and Shengmao $\mathrm{Fu}^{1}$ \\ ${ }^{1}$ College of Mathematics and Statistics, Northwest Normal University, Lanzhou 730070, China \\ ${ }^{2}$ College of Mathematics and Computer Science, Northwest University for Nationalities, Lanzhou 730124, \\ China \\ Correspondence should be addressed to Shengmao Fu, fusm@nwnu.edu.cn
}

Received 23 March 2012; Accepted 15 May 2012

Academic Editor: Michiel Bertsch

Copyright (C) 2012 Y. Jiao and S. Fu. This is an open access article distributed under the Creative Commons Attribution License, which permits unrestricted use, distribution, and reproduction in any medium, provided the original work is properly cited.

\begin{abstract}
We consider a strongly coupled predator-prey model with one resource and two consumers, in which the first consumer species feeds on the resource according to the Holling II functional response, while the second consumer species feeds on the resource following the BeddingtonDeAngelis functional response, and they compete for the common resource. Using the energy estimates and Gagliardo-Nirenberg-type inequalities, the existence and uniform boundedness of global solutions for the model are proved. Meanwhile, the sufficient conditions for global asymptotic stability of the positive equilibrium for this model are given by constructing a Lyapunov function.
\end{abstract}

\section{Introduction}

The principle of competitive exclusion asserts that two or more consumer species cannot coexist indefinitely upon a single limiting resource, which dates back to the pioneering work of Volterra [1] in the 1920s. Subsequently, Ayala [2] in 1969 demonstrated experimentally that two species of Drosophila can coexist upon a single limiting resource. Ayala's experiments have received much attention (see the comprehensive survey by Cantrell and Cosner [3]). Schoener [4] in 1976 found that intraspecific interference among consumers may lead to coexistence of multiple consumer species upon a single resource. To examine more closely the implications of feeding interference among conspecific consumers on 
consumer-resource dynamics, Cantrell et al. [5] in 2004 proposed the following predator-prey system:

$$
\begin{aligned}
& \frac{d u}{d t}=r u\left(1-\frac{u}{K}\right)-\frac{a u v}{1+b u}-\frac{A u w}{1+B u+D w}, \\
& \frac{d v}{d t}=v\left(-l+\frac{e u}{1+b u}\right) \\
& \frac{d w}{d t}=w\left(-L+\frac{E u}{1+B u+D w}\right),
\end{aligned}
$$

where $r, a, b, e, l, A, B, D, E, L$, and $K$ are positive constants, $u(t)$ represents the density of the limiting resource at time $t, v(t)$ and $w(t)$ denote two consumers species. The first consumer species feeds upon the resource according to the Holling II functional response, while the second consumer species feeds upon the resource following the Beddington-DeAngelis functional response, and they compete for the common resource. For more details on the backgrounds about this system see [5].

The system (1.1) has a positive equilibrium $\widetilde{E}=(\widetilde{u}, \widetilde{v}, \widetilde{w})$ under the suitable conditions, where

$$
\tilde{u}=\frac{l}{e-b l}, \quad \tilde{v}=\frac{e}{a l}\left\{r \tilde{u}\left(1-\frac{\tilde{u}}{K}\right)-\frac{A}{D E}[(E-B L) \tilde{u}-L]\right\}, \quad \tilde{w}=\frac{(E-B L) \tilde{u}-L}{D L} .
$$

The Jacobian matrix of the system (1.1) at $\widetilde{E}$ can be written as

$$
J(\tilde{E})=\left(\begin{array}{lll}
a_{11} & a_{12} & a_{13} \\
a_{21} & a_{22} & a_{23} \\
a_{31} & a_{32} & a_{33}
\end{array}\right),
$$

where

$$
\begin{aligned}
& a_{11}=\tilde{u}\left[-\frac{r \tilde{u}}{K}+\frac{a b \tilde{v}}{(1+b \tilde{u})^{2}}+\frac{A B \tilde{w}}{(1+B \tilde{u}+D \tilde{w})^{2}}\right], \quad a_{12}=-\frac{a \tilde{u}}{1+b \tilde{u}}<0, \\
& a_{13}=-\frac{A \tilde{u}(1+B \tilde{u})}{(1+B \tilde{u}+D \tilde{w})^{2}}<0, \quad a_{21}=\frac{e \tilde{v}}{(1+b \tilde{u})^{2}}>0, \quad a_{31}=\frac{E \tilde{w}(1+D \tilde{w})}{(1+B \tilde{u}+D \tilde{w})^{2}}>0, \\
& a_{33}=-\frac{D E \tilde{u} \tilde{w}}{(1+B \tilde{u}+D \tilde{w})^{2}}<0, \quad a_{22}=a_{23}=a_{32}=0 .
\end{aligned}
$$

The following results were proved in [5]:

(1) the system (1.1) is dissipative;

(2) the positive equilibrium $\widetilde{E}$ of $(1.1)$ is locally stable if $-\left(a_{11}+a_{33}\right)>0$ and $a_{11}\left(a_{11} a_{33}-\right.$ $\left.a_{13} a_{31}-a_{12} a_{21}\right)-a_{33}\left(a_{11} a_{33}-a_{31} a_{31}\right)<0$; and

(3) the positive equilibrium $\widetilde{E}$ of $(1.1)$ is globally stable if $\max \{b, B\}(K-\widetilde{u})<1$. 
Rescaling the system (1.1) such that

$$
\begin{aligned}
& \frac{u}{K} \longmapsto u, \quad v \longmapsto v, \quad w \longmapsto w, \quad r t \longmapsto t, \quad \frac{a}{r} \longmapsto a, \quad b K \longmapsto b, \quad \frac{A}{r} \longmapsto A, \quad B K \longmapsto B, \\
& D \longmapsto D, \quad \frac{l}{r} \longmapsto l, \quad \frac{(e K)}{l} \longmapsto e, \quad \frac{L}{r} \longmapsto L, \quad \frac{(E K)}{L} \longmapsto E
\end{aligned}
$$

yields

$$
\begin{aligned}
& \frac{d u}{d t}=u(1-u)-\frac{a u v}{1+b u}-\frac{A u w}{1+B u+D w}, \\
& \frac{d v}{d t}=l v\left(-1+\frac{e u}{1+b u}\right) \\
& \frac{d w}{d t}=L w\left(-1+\frac{E u}{1+B u+D w}\right) .
\end{aligned}
$$

The corresponding weakly coupled reaction-diffusion system for (1.6) is as follows:

$$
\begin{aligned}
& u_{t}=d_{1} \Delta u+u(1-u)-\frac{a u v}{1+b u}-\frac{A u w}{1+B u+D w}, \quad x \in \Omega, t>0, \\
& v_{t}=d_{2} \Delta v+l v\left(-1+\frac{e u}{1+b u}\right), \quad x \in \Omega, t>0, \\
& w_{t}=d_{3} \Delta w+L w\left(-1+\frac{E u}{1+B u+D w}\right), \quad x \in \Omega, t>0, \\
& \partial_{v} u=\partial_{\nu} v=\partial_{v} w=0, \quad x \in \partial \Omega, t>0, \\
& u(x, 0)=u_{0}(x), \quad v(x, 0)=v_{0}(x), \quad w(x, 0)=w_{0}(x), \quad x \in \Omega,
\end{aligned}
$$

where $\Omega \subset \mathbb{R}^{N}$ is a bounded smooth domain, $v$ is the outward unit normal vector of the boundary $\partial \Omega, \partial_{v}=\partial / \partial v$. The constants $d_{1}, d_{2}$, and $d_{3}$, called diffusion coefficients, are positive, and $u_{0}(x), v_{0}(x)$, and $w_{0}(x)$ are nonnegative functions which are not identically zero. The system (1.7) has a constant positive steady-state solution $E^{*}=\left(u^{*}, v^{*}, w^{*}\right)$ if and only if

$$
e>b, \quad E-B>e-b, \quad D E(e-b-1)-A(e-b)[(E-B)-(e-b)]>0,
$$

where

$$
\begin{aligned}
& u^{*}=\frac{1}{e-b}, \quad v^{*}=\frac{e\{D E(e-b-1)-A(e-b)[(E-B)-(e-b)]\}}{a D E(e-b)^{2}}, \\
& w^{*}=\frac{(E-B)-(e-b)}{D(e-b)} .
\end{aligned}
$$


In [6], Hei and Yu proved the following main results.

(1) The equilibrium $\left(u^{*}, v^{*}, w^{*}\right)$ of (1.7) is locally asymptotically stable if (1.8) and

$$
D E^{2}[b(e-b-1)-e]+A(e-b)(B e-b E)[(E-B)-(e-b)]<0
$$

hold.

(2) Let $d_{2}^{*}$ and $d_{3}^{*}$ be fixed positive constants which satisfy $d_{2}^{*} \mu_{1} \geq l(e-b-1) /(1+b)$ and $d_{3}^{*} \mu_{1} \geq L(E-B-1) /(1+B)$.

Then there exists a positive constant $D_{1}$, such that (1.7) has no nonconstant positive solution if $d_{1}>D_{1}, d_{2} \geq d_{2}^{*}$ and $d_{3} \geq d_{3}^{*}$, where $0=\mu_{0}<\mu_{1}<\mu_{2}<\cdots$ are the eigenvalues of the operator $-\Delta$ on $\Omega$ with the homogeneous Neumann boundary condition.

(3) Let $d_{i}(i=1,3)$ be fixed positive constants. Assume that $a_{11}^{*}>0$,

$$
\min \left\{\frac{4 d_{2}-4 e l d_{1}-e d_{2}}{4 d_{2}}, \frac{[D(E-B)-A(E-B-1)]}{D(E-B)}\right\}>\frac{1}{e-b}
$$

and (1.8) hold. Furthermore, assume that one of the following conditions is satisfied:

(i) $a_{11}^{*} a_{33}^{*}-a_{31}^{*} a_{13}^{*}<0, \check{\mu} \in\left(\mu_{n}, \mu_{n+1}\right)$ for some $n \geq 1$, and the sum $\sigma_{n}=\sum_{i=1}^{n} \operatorname{dim} E\left(\mu_{i}\right)$ is odd;

(ii) $a_{11}^{*} a_{33}^{*}-a_{31}^{*} a_{13}^{*}>0, \widehat{\mu} \in\left(\mu_{k}, \mu_{k+1}\right), \check{\mu} \in\left(\mu_{n}, \mu_{n+1}\right)$ for some $n \geq k \geq 1$, and the sum $\sigma_{n}=\sum_{i=k+1}^{n} \operatorname{dim} E\left(\mu_{i}\right)$ is odd.

Then there exists a positive constant $D_{2}$, such that (1.7) has at least one nonconstant positive solution if $d_{2} \geq D_{2}$, where

$$
\begin{aligned}
& a_{11}^{*}=-u^{*}+\frac{a b v^{*}}{e^{2} u^{*}}+\frac{A B w^{*}}{E^{2} u^{*}}, \\
& a_{33}^{*}=-\frac{D E L u^{*} w^{*}}{\left(1+B u^{*}+D w^{*}\right)^{2}}, \\
& a_{13}^{*}=-\frac{A w^{*}}{E u^{*}}, \\
& a_{31}^{*}=\frac{E L w^{*}\left(1+D w^{*}\right)}{\left(1+B u^{*}+D w^{*}\right)^{2}} \\
& \check{\mu}=\frac{a_{11}^{*} d_{3}+a_{33}^{*} d_{1}+\sqrt{\left(a_{11}^{*} d_{3}-a_{33}^{*} d_{1}\right)^{2}+4 a_{31}^{*} a_{13}^{*} d_{1} d_{3}}}{2 d_{1} d_{3}}, \\
& \widehat{\mu}=\frac{a_{11}^{*} d_{3}+a_{33}^{*} d_{1}-\sqrt{\left(a_{11}^{*} d_{3}-a_{33}^{*} d_{1}\right)^{2}+4 a_{31}^{*} a_{13}^{*} d_{1} d_{3}}}{2 d_{1} d_{3}}
\end{aligned}
$$

and $E\left(\mu_{i}\right)$ is the eigenspace corresponding to $\mu_{i}$ in $H^{1}(\Omega)$.

(4) The bifurcation of nonconstant positive solutions for (1.7) was studied.

In recent years, the SKT type cross-diffusion systems have attracted the attention of a great number of investigators and have been successfully developed on the theoretical 
backgrounds. The above work mainly concentrate on (1) the instability and stability induced by cross-diffusion, and the existence of nonconstant positive steady-state solutions [7-14]; (2) the global existence of strong solutions [15-23]; (3) the global existence of weak solutions based on semidiscretization or finite element approximation [24-30]; and (4) the dynamical behaviors [18, 19, 31, 32], and so forth. The corresponding SKT type cross-diffusion system for (1.7) is as follows:

$$
\begin{aligned}
& u_{t}=\Delta\left(d_{1} u+\alpha_{11} u^{2}+\alpha_{12} u v+\alpha_{13} u w\right)+u(1-u)-\frac{a u v}{1+b u}-\frac{A u w}{1+B u+D w}, \quad x \in \Omega, t>0, \\
& v_{t}=\Delta\left(d_{2} v+\alpha_{21} u v+\alpha_{22} v^{2}+\alpha_{23} v w\right)+l v\left(-1+\frac{e u}{1+b u}\right), \quad x \in \Omega, t>0, \\
& w_{t}=\Delta\left(d_{3} w+\alpha_{31} u w+\alpha_{32} v w+\alpha_{33} w^{2}\right)+L w\left(-1+\frac{E u}{1+B u+D w}\right), \quad x \in \Omega, t>0, \\
& \partial_{v} u=\partial_{v} v=\partial_{v} w=0, \quad x \in \partial \Omega, t>0, \\
& u(x, 0)=u_{0}(x), \quad v(x, 0)=v_{0}(x), \quad w(x, 0)=w_{0}(x), \quad x \in \Omega,
\end{aligned}
$$

where $\alpha_{i j}(i, j=1,2,3)$ are positive constants, $\alpha_{i i}(i=1,2,3)$ are referred as self-diffusion pressures, and $\alpha_{i j}(i, j=1,2,3, i \neq j)$ are cross-diffusion pressures. The self-diffusion implies the movement of individuals from a higher to lower concentration region. Cross-diffusion expresses the population fluxes of one species due to the presence of the other species. The value of cross-diffusion coefficient may be positive, negative, or zero. The positive cross-diffusion coefficient denotes the movement of the species in the direction of lower concentration of another species and negative cross-diffusion coefficient denotes that one species tends to diffuse in the direction of higher concentration of another species (e.g., [33]).

The local existence of solutions for the system (1.13) is an immediate consequence of a series of important papers [34-36] by Amann. Roughly speaking, if $u_{0}(x), v_{0}(x)$, and $w_{0}(x)$ in $W_{p}^{1}(\Omega)$ with $p>n$, then (1.13) has a unique nonnegative solution $u, v, w \in C\left([0, T), W_{p}^{1}(\Omega)\right) \cap$ $C^{\infty}\left((0, T), C^{\infty}(\Omega)\right)$, where $T \in(0, \infty]$ is the maximal existence time for the solution. If the solution $(u, v, w)$ satisfies the estimate

$$
\sup \left\{\|u(\cdot, t)\|_{W_{p}^{1}(\Omega)},\|v(\cdot, t)\|_{W_{p}^{1}(\Omega)},\|w(\cdot, t)\|_{W_{p}^{1}(\Omega)}: 0<t<T\right\}<\infty,
$$

then $T=+\infty$. Moreover, if $u_{0}(x), v_{0}(x), w_{0}(x) \in W_{p}^{2}(\Omega)$, then $u, v, w \in C\left([0, \infty), W_{p}^{2}(\Omega)\right)$.

For the following SKT system

$$
\begin{aligned}
& u_{t}=d_{1} \Delta[(1+\alpha v+\gamma u) u]+a u(1-u-c v), \quad x \in \Omega, t>0, \\
& v_{t}=d_{2} \Delta[(1+\delta v) v]+b v(1-d u-v), \quad x \in \Omega, t>0 \\
& \partial_{\nu} u=\partial_{\nu} v=0, \quad x \in \partial \Omega, t>0 \\
& u(x, 0)=u_{0}(x), \quad v(x, 0)=v_{0}(x), \quad x \in \Omega
\end{aligned}
$$


Yamada in [23] proposed four open problems:

(1) the global existence of solutions of $(\mathrm{P})$ in the case $\delta>0$ and the space dimension $N \geq 6$;

(2) the global existence in the case $\gamma=0$;

(3) in order to study the asymptotic behavior of $u, v$ as $t \rightarrow \infty$ need to establish the uniform boundedness of global solutions; and

(4) the global existence of solutions for the following full SKT system:

$$
\begin{aligned}
& u_{t}=d_{1} \Delta[(1+\alpha v+\gamma u) u]+a u(1-u-c v), \quad x \in \Omega, t>0, \\
& v_{t}=d_{2} \Delta[(1+\beta u+\delta v) v]+b v(1-d u-v), \quad x \in \Omega, t>0, \\
& \partial_{v} u=\partial_{\nu} v=0, \quad x \in \partial \Omega, t>0, \\
& u(x, 0)=u_{0}(x), \quad v(x, 0)=v_{0}(x), \quad x \in \Omega
\end{aligned}
$$

with $\alpha, \gamma, \beta, \delta>0$.

Very few global existence results for (1.13) are known. The main purpose of this paper is to establish the uniform boundedness of global solutions for the system (1.13) in one space dimension. For convenience, we consider the following system:

$$
\begin{aligned}
& u_{t}=\left(d_{1} u+\alpha_{11} u^{2}+\alpha_{12} u v+\alpha_{13} u w\right)_{x x}+u(1-u)-\frac{a u v}{1+b u}-\frac{A u w}{1+B u+D w}, \quad 0<x<1, t>0, \\
& v_{t}=\left(d_{2} v+\alpha_{21} u v+\alpha_{22} v^{2}+\alpha_{23} v w\right)_{x x}+l v\left(-1+\frac{e u}{1+b u}\right), \quad 0<x<1, t>0, \\
& w_{t}=\left(d_{3} w+\alpha_{31} u w+\alpha_{32} v w+\alpha_{33} w^{2}\right)_{x x}+L w\left(-1+\frac{E u}{1+B u+D w}\right), \quad 0<x<1, t>0, \\
& u_{x}(x, t)=v_{x}(x, t)=w_{x}(x, t)=0, \quad x=0,1, t>0, \\
& u(x, 0)=u_{0}(x), \quad v(x, 0)=v_{0}(x), \quad w(x, 0)=w_{0}(x), \quad 0<x<1 .
\end{aligned}
$$

We firstly investigate the global existence and the uniform boundedness of the solutions for (1.16), then prove the global asymptotic stability of the positive equilibrium $\left(u^{*}, v^{*}, w^{*}\right)$ of (1.16) by an important lemma from [37]. The proof is complete and complement to the uniform convergence theorems in papers [38-40]. (1.8) holds.

It is obvious that $\left(u^{*}, v^{*}, w^{*}\right)$ is the unique positive equilibrium of the system (1.16) if

For simplicity, we denote $\|\cdot\|_{W_{p}^{k}(0,1)}$ by $|\cdot|_{k, p}$ and $\|\cdot\|_{L^{p}(0,1)}$ by $|\cdot|_{p}$. Our main results are as follows. 
Theorem 1.1. Let $u_{0}(x), v_{0}(x), w_{0}(x) \in W_{2}^{2}(0,1),(u, v, w)$ be the unique nonnegative solution of the system (1.16) in the maximal existence interval $[0, T)$. Assume that

$$
\begin{aligned}
& 8 \alpha_{11} \alpha_{21} \alpha_{31}>\alpha_{21} \alpha_{13}^{2}+\alpha_{12}^{2} \alpha_{31} \\
& 8 \alpha_{12} \alpha_{22} \alpha_{32}>\alpha_{32} \alpha_{21}^{2}+\alpha_{23}^{2} \alpha_{12} \\
& 8 \alpha_{13} \alpha_{23} \alpha_{33}>\alpha_{23} \alpha_{31}^{2}+\alpha_{32}^{2} \alpha_{13}
\end{aligned}
$$

Then there exist $t_{0}>0$ and positive constants $M^{\prime}, M$ which depend on $d_{i}, \alpha_{i j}(i, j=$ $1,2,3), a, b, e, l, A, B, D, E$ and $L$, such that

$$
\begin{gathered}
\sup \left\{|u(\cdot, t)|_{1,2},|v(\cdot, t)|_{1,2},|w(\cdot, t)|_{1,2}: t \in\left(t_{0}, T\right)\right\} \leq M^{\prime}, \\
\max \left\{u(x, t), v(x, t), w(x, t):(x, t) \in[0,1] \times\left(t_{0}, T\right)\right\} \leq M,
\end{gathered}
$$

and $T=+\infty$. Moreover, in the case that $d_{1}, d_{2}, d_{3} \geq 1, d_{2} / d_{1}, d_{3} / d_{1} \in[\underline{d}, \bar{d}]$, where $\underline{d}$ and $\bar{d}$ are positive constants, $M^{\prime}, M$ depend on $\underline{d}, \bar{d}$, but do not depend on $d_{1}, d_{2}, d_{3}$.

Remark 1.2. Since the continuous embedding $H^{1}(\Omega) \hookrightarrow L^{\infty}(\Omega)$ holds only in one space dimension, we can only establish the uniform maximum-norn estimates about time for the solution in one space dimension.

Theorem 1.3. Assume that all conditions in Theorem 1.1 are satisfied. Assume further that

$$
\begin{aligned}
4 \alpha \beta u^{*} v^{*} w^{*} d_{1} d_{2} d_{3}> & u^{*} M^{2}\left(\alpha \alpha_{23} v^{*}+\beta \alpha_{32} w^{*}\right)^{2}\left(d_{1}+2 \alpha_{11} M+\alpha_{12} M+\alpha_{13} M\right) \\
& +\alpha v^{*} M^{2}\left(\alpha_{13} u^{*}+\beta \alpha_{31} w^{*}\right)^{2}\left(d_{2}+\alpha_{21} M+2 \alpha_{22} M+\alpha_{23} M\right) \\
& +\beta w^{*} M^{2}\left(\alpha_{12} u^{*}+\alpha \alpha_{21} v^{*}\right)^{2}\left(d_{3}+\alpha_{31} M+\alpha_{32} M+2 \alpha_{33} M\right), \\
D E[(e-b) & -b(e-b-1)]>A(e-b)[(E-B)-(e-b)](B-b),
\end{aligned}
$$

and (1.8) hold, where $\alpha=a\left(1+b u^{*}\right) / e l, \beta=A\left(1+B u^{*}\right) / E L\left(1+D w^{*}\right), M$ is given by (1.19). Then the unique positive equilibrium $\left(u^{*}, v^{*}, w^{*}\right)$ of $(1.16)$ is globally asymptotically stable.

Remark 1.4. The system (1.16) has no nonconstant positive steady-state solution if all conditions of Theorem 1.3 hold. 
Examples. The following two examples satisfy all conditions of Theorem 1.3:

$$
\begin{aligned}
& \alpha_{11}=\frac{1}{4}, \quad \alpha_{12}=1, \quad \alpha_{13}=1, \\
& \alpha_{21}=\frac{5}{2}, \quad \alpha_{22}=1, \quad \alpha_{23}=\frac{1}{4}, \\
& \alpha_{31}=1, \quad \alpha_{32}=1, \quad \alpha_{33}=2, \\
& b=1, \quad e=3, \quad A=1, \\
& B=\frac{1}{2}, \quad D=\frac{2}{3}, \quad E=3, \\
& d_{1}, d_{2}, d_{3} \gg 1 ; \\
& \alpha_{11}=2, \quad \alpha_{12}=1, \quad \alpha_{13}=2, \\
& \alpha_{21}=\frac{3}{2}, \quad \alpha_{22}=\frac{1}{2}, \quad \alpha_{23}=1, \\
& \alpha_{31}=2, \quad \alpha_{32}=2, \quad \alpha_{33}=3, \\
& b=\frac{1}{2}, \quad e=\frac{5}{2}, \quad A=\frac{2}{3}, \\
& B=\frac{1}{2}, \quad D=\frac{3}{4}, \quad E=4, \\
& d_{1}, d_{2}, d_{3} \gg 1 . \quad
\end{aligned}
$$

\section{Global Solutions}

In order to establish the uniform $W_{2}^{1}$-estimates of the solutions for the system (1.16), the following Gagliardo-Nirenberg-type inequalities and the corresponding corollary play important roles (see $[38,41])$.

Theorem 2.1. Let $\Omega \subset \mathbb{R}^{n}$ be a bounded domain with $\partial \Omega \in C^{m}$. For every function $u \in W_{r}^{m}(\Omega), 1 \leq$ $q, r \leq \infty$, the derivative $D^{j} u \quad(0 \leq j<m)$ satisfies the inequality

$$
\left|D^{j} u\right|_{p} \leq C\left(\left|D^{m} u\right|_{r}^{a}|u|_{q}^{1-a}+|u|_{q}\right)
$$

provided one of the following three conditions is satisfied: (1) $r \leq q$, (2) $0<n(r-q) /(m r q)<1$, or (3) $n(r-q) /(m r q)=1$ and $m-n / q$ is not a nonnegative integer, where $1 / p=j / n+a(1 / r-$ $m / n)+(1-a) / q$ for all $a \in[j / m, 1)$, and the positive constant $C$ depends on $n, m, j, q, r, a$. 
Corollary 2.2. There exists a universal constant $C$ such that

$$
\begin{aligned}
&|u|_{2} \leq C\left(\left|u_{x}\right|_{2}^{1 / 3}|u|_{1}^{2 / 3}+|u|_{1}\right), \forall u \in W_{2}^{1}(0,1), \\
&|u|_{4} \leq C\left(\left|u_{x}\right|_{2}^{1 / 2}|u|_{1}^{1 / 2}+|u|_{1}\right), \quad \forall u \in W_{2}^{1}(0,1), \\
&|u|_{5 / 2} \leq C\left(\left|u_{x}\right|_{2}^{2 / 5}|u|_{1}^{3 / 5}+|u|_{1}\right), \quad \forall u \in W_{2}^{1}(0,1), \\
&\left|u_{x}\right|_{2} \leq C\left(\left|u_{x x}\right|_{2}^{3 / 5}|u|_{1}^{2 / 5}+|u|_{1}\right), \quad \forall u \in W_{2}^{2}(0,1) .
\end{aligned}
$$

Throughout this paper, we always denote that $C$ is a Sobolev embedding constant or other kind of universal constant, $A_{j}, B_{j}, C_{j}$ are some positive constants which depend only on $\alpha_{i j}(i, j=1,2,3), a, b, e, l, A, B, D, E$ and $L, K$ are positive constants depending on $d_{i}, \alpha_{i j}(i, j=$ $1,2,3), a, b, e, l, A, B, D, E$ and L. When $d_{1}, d_{2}, d_{3} \geq 1, d_{2} / d_{1}, d_{3} / d_{1} \in[\underline{d}, \bar{d}], K_{j}$ depend on $\underline{d}, \bar{d}$, but do not depend on $d_{1}, d_{2}, d_{3}$.

Proof of Theorem 1.1. Taking integration of the three equations in (1.16) over $(0,1)$, respectively, and combining the three integration equalities linearly, we have

$$
\frac{d}{d t} \int_{0}^{1}[(e l+E L) u+a v+A w] d x \leq \int_{0}^{1}[(e l+E L) u(1-u)-a l v-A L w] d x
$$

It follows from the Young inequality and the Hölder inequality that

$$
\frac{d}{d t} \int_{0}^{1}[(e l+E L) u+a v+A w] d x \leq C_{1}-k \int_{0}^{1}[(e l+E L) u+a v+A w] d x
$$

where $k=\min \{l, L\}, C_{1}=(k+1)^{2}(e l+E L) / 4$. So there exist positive constants $M_{0}$ and $\tau_{0}$ depending on $a, b, e, l, A, B, D, E$, and $L$, such that

$$
\int_{0}^{1} u d x, \int_{0}^{1} v d x, \int_{0}^{1} w d x \leq M_{0}, \quad t \geq \tau_{0}
$$

Moreover, there exists a positive constant $M_{0}^{\prime}$ which depends on $a, b, e, l, A, B, D, E, L$ and $L^{1}$-norm of $u_{0}, v_{0}, w_{0}$, such that

$$
\int_{0}^{1} u d x, \int_{0}^{1} v d x, \int_{0}^{1} w d x \leq M_{0}^{\prime}, \quad t \geq 0
$$


Multiplying the first three equations in the system (1.16) by $u, v, w$, respectively, and integrating over $(0,1)$, we have

$$
\begin{aligned}
\frac{1}{2} \frac{d}{d t} \int_{0}^{1} u^{2} d x \leq & -d_{1} \int_{0}^{1} u_{x}^{2} d x-\int_{0}^{1}\left[\left(2 \alpha_{11} u+\alpha_{12} v+\alpha_{13} w\right) u_{x}^{2}+\left(\alpha_{12} v_{x}+\alpha_{13} w_{x}\right) u u_{x}\right] d x \\
& +\int_{0}^{1} u^{2} d x
\end{aligned}
$$

$$
\begin{aligned}
\frac{1}{2} \frac{d}{d t} \int_{0}^{1} v^{2} d x \leq & -d_{2} \int_{0}^{1} v_{x}^{2} d x-\int_{0}^{1}\left[\left(\alpha_{21} u+2 \alpha_{22} v+\alpha_{23} w\right) v_{x}^{2}+\alpha_{21}\left(u_{x}+\alpha_{23} w_{x}\right) v v_{x}\right] d x \\
& +\frac{e l}{b} \int_{0}^{1} v^{2} d x
\end{aligned}
$$

$$
\begin{aligned}
\frac{1}{2} \frac{d}{d t} \int_{0}^{1} w^{2} d x \leq & -d_{3} \int_{0}^{1} w_{x}^{2} d x-\int_{0}^{1}\left[\left(\alpha_{31} u+\alpha_{32} v+2 \alpha_{33} w\right) w_{x}^{2}+\left(\alpha_{31} u_{x}+\alpha_{32} v_{x}\right) w w_{x}\right] d x \\
& +\frac{E L}{B} \int_{0}^{1} w^{2} d x
\end{aligned}
$$

from which it follows that

$$
\begin{aligned}
& \frac{1}{2} \frac{d}{d t} \int_{0}^{1}\left(u^{2}+v^{2}+w^{2}\right) d x \\
& \quad \leq-d \int_{0}^{1}\left(u_{x}^{2}+v_{x}^{2}+w_{x}^{2}\right) d x+\int_{0}^{1} u^{2} d x+\frac{e l}{b} \int_{0}^{1} v^{2} d x+\frac{E L}{B} \int_{0}^{1} w^{2} d x-\int_{0}^{1} q\left(u_{x}, v_{x}, w_{x}\right) d x \\
& \quad \leq-d \int_{0}^{1}\left(u_{x}^{2}+v_{x}^{2}+w_{x}^{2}\right) d x+\left(1+\frac{e l}{b}+\frac{E L}{B}\right) \int_{0}^{1}\left(u^{2}+v^{2}+w^{2}\right) d x-\int_{0}^{1} q\left(u_{x}, v_{x}, w_{x}\right) d x
\end{aligned}
$$

where $d=\min \left\{d_{1}, d_{2}, d_{3}\right\}$,

$$
\begin{aligned}
q\left(u_{x}, v_{x}, w_{x}\right)= & \left(2 \alpha_{11} u+\alpha_{12} v+\alpha_{13} w\right) u_{x}^{2}+\left(\alpha_{12} u+\alpha_{21} v\right) u_{x} v_{x}+\left(\alpha_{21} u+2 \alpha_{22} v+\alpha_{23} w\right) v_{x}^{2} \\
& +\left(\alpha_{13} u+\alpha_{31} w\right) u_{x} w_{x}+\left(\alpha_{31} u+\alpha_{32} v+2 \alpha_{33} w\right) w_{x}^{2}+\left(\alpha_{23} v+\alpha_{32} w\right) v_{x} w_{x}
\end{aligned}
$$

It is obvious that $q\left(u_{x}, v_{x}, w_{x}\right)$ is a positive definite quadratic form of $u_{x}, v_{x}, w_{x}$ if (1.17) holds. So (1.17) implies that

$$
\frac{1}{2} \frac{d}{d t} \int_{0}^{1}\left(u^{2}+v^{2}+w^{2}\right) d x \leq-d \int_{0}^{1}\left(u_{x}^{2}+v_{x}^{2}+w_{x}^{2}\right) d x+\left(1+\frac{e l}{b}+\frac{E L}{B}\right) \int_{0}^{1}\left(u^{2}+v^{2}+w^{2}\right) d x .
$$


Now, we proceed in the following two cases.

(i) One has $t \geq \tau_{0}$. The inequality (2.2) implies that $|u|_{2}^{6} \leq C\left(\left|u_{x}\right|_{2}^{2}|u|_{1}^{4}+|u|_{1}^{6}\right) \leq$ $C M_{0}^{4}\left(\left|u_{x}\right|_{2}^{2}+M_{0}^{2}\right)$. So we have $\int_{0}^{1} u_{x}^{2} d x \geq\left(1 / C M_{0}^{4}\right)\left(\int_{0}^{1} u^{2} d x\right)^{3}-M_{0}^{2}$, and

$$
-\int_{0}^{1}\left(u_{x}^{2}+v_{x}^{2}+w_{x}^{2}\right) d x \leq-\frac{1}{9 C M_{0}^{4}}\left[\int_{0}^{1}\left(u^{2}+v^{2}+w^{2}\right) d x\right]^{3}+3 M_{0}^{2}
$$

It follows from (2.12) and (2.13) that

$$
\begin{aligned}
& \frac{1}{2} \frac{d}{d t} \int_{0}^{1}\left(u^{2}+v^{2}+w^{2}\right) d x \\
& \quad \leq d\left\{-C_{2}\left[\int_{0}^{1}\left(u^{2}+v^{2}+w^{2}\right) d x\right]^{3}+3 M_{0}^{2}+\frac{1}{d}\left(1+\frac{e l}{b}+\frac{E L}{B}\right) \int_{0}^{1}\left(u^{2}+v^{2}+w^{2}\right) d x\right\} .
\end{aligned}
$$

This means that there exist positive constants $\tau_{1}$ and $M_{1}$ depending on $d_{i}(i=$ $1,2,3), a, b, e, l, A, B, D, E$, and $L$, such that

$$
\int_{0}^{1} u^{2} d x, \int_{0}^{1} v^{2} d x, \int_{0}^{1} w^{2} d x \leq M_{1}, \quad t \geq \tau_{1}
$$

When $d \geq 1, M_{1}$ is independent of $d$ because the zero point of the right-hand side in (2.14) can be estimated by positive constants independent of $d$.

(ii) One has $t \geq 0$. Repeating estimates in (i) by $(2.8)^{\prime}$, we can obtain that there exists a positive constant $M_{1}^{\prime}$ depending on $d_{i}(i=1,2,3), a, b, e, l, A, B, D, E, L$ and the $L^{1}, L^{2}$-norm of $u_{0}, v_{0}, w_{0}$, such that

$$
\int_{0}^{1} u^{2} d x, \int_{0}^{1} v^{2} d x, \int_{0}^{1} w^{2} d x \leq M_{1}^{\prime}, \quad t \geq 0
$$

When $d \geq 1, M_{1}^{\prime}$ is independent of $d$.

To estimate $\left|u_{x}\right|_{2},\left|v_{x}\right|_{2},\left|w_{x}\right|_{2}$, we introduce the following scaling:

$$
\widehat{u}=\frac{u}{d_{1}}, \quad \widehat{v}=\frac{v}{d_{1}}, \quad \widehat{w}=\frac{w}{d_{1}}, \quad \widehat{t}=d_{1} t
$$


Denoting $\xi=d_{2} / d_{1}, \eta=d_{3} / d_{1}$, and using $u, v, w, t$ instead of $\widehat{u}, \widehat{v}, \widehat{w}, \widehat{t}$, respectively, then the system (1.16) reduces to

$$
\begin{aligned}
& u_{t}=P_{x x}+f(u, v, w), \quad 0<x<1, t>0, \\
& v_{t}=Q_{x x}+g(u, v, w), \quad 0<x<1, t>0, \\
& w_{t}=R_{x x}+h(u, v, w), \quad 0<x<1, t>0, \\
& u_{x}(x, t)=v_{x}(x, t)=w_{x}(x, t)=0, \quad x=0,1, t>0, \\
& u(x, 0)=\widehat{u}_{0}(x), \quad v(x, 0)=\widehat{v}_{0}(x), \quad w(x, 0)=\widehat{w}_{0}(x), \quad 0<x<1,
\end{aligned}
$$

where $P=u+\alpha_{11} u^{2}+\alpha_{12} u v+\alpha_{13} u w, Q=\xi v+\alpha_{21} u v+\alpha_{22} v^{2}+\alpha_{23} v w, R=\eta w+\alpha_{31} u w+\alpha_{32} v w+$ $\alpha_{33} w^{2}, f(u, v, w)=d_{1}^{-1} u-u^{2}-\left(a u v /\left(1+b d_{1} u\right)\right)-\left(A u w /\left(1+B d_{1} u+D d_{1} w\right)\right), g(u, v, w)=$ $l v\left(-d_{1}^{-1}+\left(e u /\left(1+b d_{1} u\right)\right)\right), h(u, v, w)=L w\left(-d_{1}^{-1}+\left(E u /\left(1+B d_{1} u+D d_{1} w\right)\right)\right)$.

We still proceed in the following two cases.

(i) One has $t \geq \tau_{1}^{*}=d_{1} \tau_{1}$. It is not hard to verify that

$$
\begin{aligned}
& \int_{0}^{1} u d x, \int_{0}^{1} v d x, \int_{0}^{1} w d x \leq M_{0} d_{1}^{-1}, \\
& \int_{0}^{1} u^{2} d x, \int_{0}^{1} v^{2} d x, \int_{0}^{1} w^{2} d x \leq M_{1} d_{1}^{-2}, \\
& |P|_{1},|Q|_{1},|R|_{1} \leq A_{1} K_{1} d_{1}^{-1},
\end{aligned}
$$

where $K_{1}=(1+\xi+\eta)+M_{1} d_{1}^{-1}, A_{1}=\max \left\{M_{0}, \alpha_{11}+\alpha_{12}+\alpha_{13}, \alpha_{21}+\alpha_{22}+\alpha_{23}, \alpha_{31}+\alpha_{32}+\alpha_{33}\right\}$. Multiplying the first three equations in (2.17) by $P_{t}, Q_{t}, R_{t}$, integrating them over the domain $(0,1)$, respectively, and then adding up the three integration equalities, we have

$$
\begin{aligned}
\frac{1}{2} \bar{y}^{\prime}(t)= & -\int_{0}^{1} u_{t}^{2} d x-\xi \int_{0}^{1} v_{t}^{2} d x-\eta \int_{0}^{1} w_{t}^{2} d x-\int_{0}^{1} q\left(u_{t}, v_{t}, w_{t}\right) d x \\
& +\int_{0}^{1}\left[\left(1+2 \alpha_{11} u+\alpha_{12} v+\alpha_{13} w\right) u_{t} f+\alpha_{12} u v_{t} f+\alpha_{13} u w_{t} f\right] d x \\
& +\int_{0}^{1}\left[\left(\xi+\alpha_{21} u+2 \alpha_{22} v+\alpha_{23} w\right) v_{t} g+\alpha_{21} v u_{t} g+\alpha_{23} v w_{t} g\right] d x \\
& +\int_{0}^{1}\left[\left(\eta+\alpha_{31} u+\alpha_{32} v+2 \alpha_{33} w\right) w_{t} h+\alpha_{31} w u_{t} h+\alpha_{32} w v_{t} h\right] d x,
\end{aligned}
$$

where $\bar{y}(t)=\int_{0}^{1}\left(P_{x}^{2}+Q_{x}^{2}+R_{x}^{2}\right) d x$. Notice by (1.17) that there exists a positive constant $C_{3}$ depending only on $\alpha_{i j}(i, j=1,2,3)$, such that

$$
q\left(u_{t}, v_{t}, w_{t}\right) \geq C_{3}(u+v+w)\left(u_{t}^{2}+v_{t}^{2}+w_{t}^{2}\right)
$$


Thus,

$$
\begin{aligned}
\frac{1}{2} \bar{y}^{\prime}(t) \leq & -\int_{0}^{1} u_{t}^{2} d x-\xi \int_{0}^{1} v_{t}^{2} d x-\eta \int_{0}^{1} w_{t}^{2} d x-C_{3} \int_{0}^{1}(u+v+w)\left(u_{t}^{2}+v_{t}^{2}+w_{t}^{2}\right) d x \\
& +\int_{0}^{1}\left[\left(1+2 \alpha_{11} u+\alpha_{12} v+\alpha_{13} w\right) u_{t} f+\alpha_{12} u v_{t} f+\alpha_{13} u w_{t} f\right] d x \\
& +\int_{0}^{1}\left[\left(\xi+\alpha_{21} u+2 \alpha_{22} v+\alpha_{23} w\right) v_{t} g+\alpha_{21} v u_{t} g+\alpha_{23} v w_{t} g\right] d x \\
& +\int_{0}^{1}\left[\left(\eta+\alpha_{31} u+\alpha_{32} v+2 \alpha_{33} w\right) w_{t} h+\alpha_{31} w u_{t} h+\alpha_{32} w v_{t} h\right] d x .
\end{aligned}
$$
estimates:

Using the Young inequality, Hölder inequality, and (2.18), we can obtain the following

$$
\begin{aligned}
& \int_{0}^{1} u^{4} d x \leq\left(\int_{0}^{1} u^{2} d x\right)^{1 / 3}\left(\int_{0}^{1} u^{5} d x\right)^{2 / 3} \leq M_{1}^{1 / 3} d_{1}^{-2 / 3}\left(\int_{0}^{1} u^{5} d x\right)^{2 / 3}, \\
& \int_{0}^{1} u v^{2} d x \leq\left(\int_{0}^{1} u^{2} d x\right)^{1 / 2}\left(\int_{0}^{1} v^{2} d x\right)^{1 / 6}\left(\int_{0}^{1} v^{5} d x\right)^{1 / 3} \leq M_{1}^{2 / 3} d_{1}^{-4 / 3}\left(\int_{0}^{1} v^{5} d x\right)^{1 / 3}, \\
& \int_{0}^{1} u^{3} d x \leq\left(\int_{0}^{1} u^{2} d x\right)^{2 / 3}\left(\int_{0}^{1} u^{5} d x\right)^{1 / 3} \leq M_{1}^{2 / 3} d_{1}^{-4 / 3}\left(\int_{0}^{1} u^{5} d x\right)^{1 / 3}, \\
& \int_{0}^{1} u^{4} v d x \leq \frac{4}{5} \int_{0}^{1} u^{5} d x+\frac{1}{5} \int_{0}^{1} v^{5} d x \leq \frac{4}{5} \int_{0}^{1}\left(u^{5}+v^{5}\right) d x .
\end{aligned}
$$

Applying the above estimates and Gagliardo-Nirenberg-type inequalities to the terms on the right-hand side of (2.21), we have

$$
\begin{aligned}
& -\int_{0}^{1} u_{t}^{2} d x \leq-\frac{1}{2} \int_{0}^{1} P_{x x}^{2} d x+\int_{0}^{1} f^{2} d x, \\
& -\xi \int_{0}^{1} v_{t}^{2} d x \leq-\frac{\xi}{2} \int_{0}^{1} Q_{x x}^{2} d x+\xi \int_{0}^{1} g^{2} d x, \\
& -\eta \int_{0}^{1} w_{t}^{2} d x \leq-\frac{\eta}{2} \int_{0}^{1} R_{x x}^{2} d x+\eta \int_{0}^{1} h^{2} d x,
\end{aligned}
$$




$$
\begin{aligned}
& \int_{0}^{1} f^{2} d x \leq \int_{0}^{1}\left(d_{1}^{-2} u^{2}+u^{4}+\frac{a^{2} v^{2}}{\left(b d_{1}\right)^{2}}+\frac{A^{2} w^{2}}{\left(B d_{1}\right)^{2}}+2 \frac{a u^{2} v}{b d_{1}}+2 \frac{A u^{2} w}{B d_{1}}+2 \frac{a A v w}{b B d_{1}^{2}}\right) d x \\
& \leq {\left[1+2\left(\frac{a}{b}\right)^{2}+2\left(\frac{A}{B}\right)^{2}\right] M_{1} d_{1}^{-4}+M_{1}^{1 / 3} d_{1}^{-2 / 3}\left(\int_{0}^{1} u^{5} d x\right)^{2 / 3} } \\
&+2\left(\frac{a}{b}+\frac{A}{B}\right) M_{1}^{2 / 3} d_{1}^{-7 / 3}\left(\int_{0}^{1} u^{5} d x\right)^{1 / 3} \\
& \xi \int_{0}^{1} g^{2} d x \leq \xi \int_{0}^{1}\left(l^{2} d_{1}^{-2} v^{2}+\frac{e^{2} l^{2} v^{2}}{\left(b d_{1}\right)^{2}}\right) d x \leq \xi l^{2}\left[1+\left(\frac{e}{b}\right)^{2}\right] M_{1} d_{1}^{-4} \\
& \eta \int_{0}^{1} h^{2} d x \leq \eta \int_{0}^{1}\left(L^{2} d_{1}^{-2} w^{2}+\frac{E^{2} L^{2} w^{2}}{\left(B d_{1}\right)^{2}}\right) d x \leq \eta L^{2}\left[1+\left(\frac{E}{B}\right)^{2}\right] M_{1} d_{1}^{-4} \\
&-\int_{0}^{1} u_{t}^{2} d x-\xi \int_{0}^{1} v_{t}^{2} d x-\eta \int_{0}^{1} w_{t}^{2} d x \\
& \leq-\frac{1}{2} \int_{0}^{1} P_{x x}^{2} d x-\frac{\xi}{2} \int_{0}^{1} Q_{x x}^{2} d x-\frac{\eta}{2} \int_{0}^{1} R_{x x}^{2} d x \\
&+\left\{1+2\left[\left(\frac{a}{b}\right)^{2}+\left(\frac{A}{B}\right)^{2}\right]+\xi l^{2}\left[1+\left(\frac{e}{b}\right)^{2}\right]+\eta L^{2}\left[1+\left(\frac{E}{B}\right)^{2}\right]\right\} M_{1} d_{1}^{-4} \\
&+M_{1}^{1 / 3} d_{1}^{-2 / 3}\left(\int_{0}^{1} u^{5} d x\right)^{2 / 3}+2\left(\frac{a}{b}+\frac{A}{B}\right) M_{1}^{2 / 3} d_{1}^{-7 / 3}\left(\int_{0}^{1} u^{5} d x\right)^{1 / 3}
\end{aligned}
$$

Similarly, we can obtain

$$
\begin{aligned}
\int_{0}^{1} u_{t} f d x \leq & \int_{0}^{1} u_{t}\left(d_{1}^{-1} u+u^{2}+\frac{a}{b d_{1}} v+\frac{A}{B d_{1}} w\right) d x \\
\leq & \frac{d_{1}^{-2}}{2 \varepsilon} \int_{0}^{1} u d x+\frac{\varepsilon}{2} \int_{0}^{1} u u_{t}^{2} d x+\frac{1}{2 \varepsilon} \int_{0}^{1} u^{3} d x+\frac{\varepsilon}{2} \int_{0}^{1} u u_{t}^{2} d x \\
& +\frac{\left(a / b d_{1}\right)^{2}}{2 \varepsilon} \int_{0}^{1} v d x+\frac{\varepsilon}{2} \int_{0}^{1} v u_{t}^{2} d x+\frac{\left(A / B d_{1}\right)^{2}}{2 \varepsilon} \int_{0}^{1} w d x+\frac{\varepsilon}{2} \int_{0}^{1} w u_{t}^{2} d x \\
\leq & \frac{1}{2 \varepsilon}\left[1+\left(\frac{a}{b}\right)^{2}+\left(\frac{A}{B}\right)^{2}\right] M_{0} d_{1}^{-3}+\frac{1}{2 \varepsilon} M_{1}^{2 / 3} d_{1}^{-4 / 3}\left(\int_{0}^{1} u^{5} d x\right)^{1 / 3} \\
& +\varepsilon \int_{0}^{1} u u_{t}^{2} d x+\frac{\varepsilon}{2} \int_{0}^{1} v u_{t}^{2} d x+\frac{\varepsilon}{2} \int_{0}^{1} w u_{t}^{2} d x
\end{aligned}
$$




$$
\begin{aligned}
& 2 \alpha_{11} \int_{0}^{1} u u_{t} f d x \leq 2 \alpha_{11} \int_{0}^{1} u u_{t}\left(d_{1}^{-1} u+u^{2}+\frac{a}{b d_{1}} v+\frac{A}{B d_{1}} w\right) d x \\
& \leq \frac{\alpha_{11}^{2} d_{1}^{-2}}{\varepsilon} \int_{0}^{1} u^{3} d x+\varepsilon \int_{0}^{1} u u_{t}^{2} d x+\frac{\alpha_{11}^{2}}{\varepsilon} \int_{0}^{1} u^{5} d x+\varepsilon \int_{0}^{1} u u_{t}^{2} d x \\
& +\frac{\alpha_{11}^{2} a^{2}}{\varepsilon b^{2} d_{1}^{2}} \int_{0}^{1} u^{2} v d x+\varepsilon \int_{0}^{1} v u_{t}^{2} d x+\frac{\alpha_{11}^{2} A^{2}}{\varepsilon B^{2} d_{1}^{2}} \int_{0}^{1} u^{2} w d x+\varepsilon \int_{0}^{1} w u_{t}^{2} d x \\
& \leq \frac{\alpha_{11}^{2}}{\varepsilon}\left[1+\left(\frac{a}{b}\right)^{2}+\left(\frac{A}{B}\right)^{2}\right] M_{1}^{2 / 3} d_{1}^{-10 / 3}\left(\int_{0}^{1} u^{5} d x\right)^{1 / 3}+\frac{\alpha_{11}^{2}}{\varepsilon} \int_{0}^{1} u^{5} d x \\
& +2 \varepsilon \int_{0}^{1} u u_{t}^{2} d x+\varepsilon \int_{0}^{1} v u_{t}^{2} d x+\varepsilon \int_{0}^{1} w u_{t}^{2} d x, \\
& \alpha_{12} \int_{0}^{1} v u_{t} f d x \leq \alpha_{12} \int_{0}^{1} v u_{t}\left(d_{1}^{-1} u+u^{2}+\frac{a}{b d_{1}} v+\frac{A}{B d_{1}} w\right) d x \\
& \leq \frac{\alpha_{12}^{2} d_{1}^{-2}}{2 \varepsilon} \int_{0}^{1} u v^{2} d x+\frac{\varepsilon}{2} \int_{0}^{1} u u_{t}^{2} d x+\frac{\alpha_{12}^{2}}{2 \varepsilon} \int_{0}^{1} u^{4} v d x+\frac{\varepsilon}{2} \int_{0}^{1} v u_{t}^{2} d x \\
& +\frac{\alpha_{12}^{2} a^{2}}{2 \varepsilon b^{2} d_{1}^{2}} \int_{0}^{1} v^{3} d x+\frac{\varepsilon}{2} \int_{0}^{1} v u_{t}^{2} d x+\frac{\alpha_{12}^{2} A^{2}}{2 \varepsilon B^{2} d_{1}^{2}} \int_{0}^{1} v^{2} w d x+\frac{\varepsilon}{2} \int_{0}^{1} w u_{t}^{2} d x \\
& \leq \frac{\alpha_{12}^{2}}{2 \varepsilon}\left[1+\left(\frac{a}{b}\right)^{2}+\left(\frac{A}{B}\right)^{2}\right] M_{1}^{2 / 3} d_{1}^{-10 / 3}\left(\int_{0}^{1} v^{5} d x\right)^{1 / 3}+\frac{2 \alpha_{12}^{2}}{5 \varepsilon} \int_{0}^{1}\left(u^{5}+v^{5}\right) d x \\
& +\frac{\varepsilon}{2} \int_{0}^{1} u u_{t}^{2} d x+\varepsilon \int_{0}^{1} v u_{t}^{2} d x+\frac{\varepsilon}{2} \int_{0}^{1} w u_{t}^{2} d x \\
& \alpha_{13} \int_{0}^{1} w u_{t} f d x \leq \alpha_{13} \int_{0}^{1} w u_{t}\left(d_{1}^{-1} u+u^{2}+\frac{a}{b d_{1}} v+\frac{A}{B d_{1}} w\right) d x \\
& \leq \frac{\alpha_{13}^{2} d_{1}^{-2}}{2 \varepsilon} \int_{0}^{1} u w^{2} d x+\frac{\varepsilon}{2} \int_{0}^{1} u u_{t}^{2} d x+\frac{\alpha_{13}^{2}}{2 \varepsilon} \int_{0}^{1} u^{4} w d x+\frac{\varepsilon}{2} \int_{0}^{1} w u_{t}^{2} d x \\
& +\frac{\alpha_{13}^{2} a^{2}}{2 \varepsilon b^{2} d_{1}^{2}} \int_{0}^{1} w^{2} v d x+\frac{\varepsilon}{2} \int_{0}^{1} v u_{t}^{2} d x+\frac{\alpha_{13}^{2} A^{2}}{2 \varepsilon B^{2} d_{1}^{2}} \int_{0}^{1} w^{3} d x+\frac{\varepsilon}{2} \int_{0}^{1} w u_{t}^{2} d x \\
& \leq \frac{\alpha_{13}^{2}}{2 \varepsilon}\left[1+\left(\frac{a}{b}\right)^{2}+\left(\frac{A}{B}\right)^{2}\right] M_{1}^{2 / 3} d_{1}^{-10 / 3}\left(\int_{0}^{1} w^{5} d x\right)^{1 / 3}+\frac{2 \alpha_{13}^{2}}{5 \varepsilon} \int_{0}^{1}\left(u^{5}+w^{5}\right) d x \\
& +\frac{\varepsilon}{2} \int_{0}^{1} u u_{t}^{2} d x+\varepsilon \int_{0}^{1} w u_{t}^{2} d x+\frac{\varepsilon}{2} \int_{0}^{1} v u_{t}^{2} d x
\end{aligned}
$$


16

Abstract and Applied Analysis

$$
\begin{aligned}
& \alpha_{12} \int_{0}^{1} u v_{t} f d x \leq \alpha_{12} \int_{0}^{1} u v_{t}\left(d_{1}^{-1} u+u^{2}+\frac{a}{b d_{1}} v+\frac{A}{B d_{1}} w\right) d x \\
& \leq \frac{\alpha_{12}^{2} d_{1}^{-2}}{2 \varepsilon} \int_{0}^{1} u^{3} d x+\frac{\varepsilon}{2} \int_{0}^{1} u v_{t}^{2} d x+\frac{\alpha_{12}^{2}}{2 \varepsilon} \int_{0}^{1} u^{5} d x+\frac{\varepsilon}{2} \int_{0}^{1} u v_{t}^{2} d x \\
& +\frac{\alpha_{12}^{2} a^{2}}{2 \varepsilon b^{2} d_{1}^{2}} \int_{0}^{1} u^{2} v d x+\frac{\varepsilon}{2} \int_{0}^{1} v v_{t}^{2} d x+\frac{\alpha_{12}^{2} A^{2}}{2 \varepsilon B^{2} d_{1}^{2}} \int_{0}^{1} u^{2} w d x+\frac{\varepsilon}{2} \int_{0}^{1} w v_{t}^{2} d x \\
& \leq \frac{\alpha_{12}^{2}}{2 \varepsilon}\left[1+\left(\frac{a}{b}\right)^{2}+\left(\frac{A}{B}\right)^{2}\right] M_{1}^{2 / 3} d_{1}^{-10 / 3}\left(\int_{0}^{1} u^{5} d x\right)^{1 / 3}+\frac{\alpha_{12}^{2}}{2 \varepsilon} \int_{0}^{1} u^{5} d x \\
& +\varepsilon \int_{0}^{1} u v_{t}^{2} d x+\frac{\varepsilon}{2} \int_{0}^{1} v v_{t}^{2} d x+\frac{\varepsilon}{2} \int_{0}^{1} w v_{t}^{2} d x, \\
& \alpha_{13} \int_{0}^{1} u w_{t} f d x \leq \alpha_{13} \int_{0}^{1} u w_{t}\left(d_{1}^{-1} u+u^{2}+\frac{a}{b d_{1}} v+\frac{A}{B d_{1}} w\right) d x \\
& \leq \frac{\alpha_{13}^{2} d_{1}^{-2}}{2 \varepsilon} \int_{0}^{1} u^{3} d x+\frac{\varepsilon}{2} \int_{0}^{1} u w_{t}^{2} d x+\frac{\alpha_{13}^{2}}{2 \varepsilon} \int_{0}^{1} u^{5} d x+\frac{\varepsilon}{2} \int_{0}^{1} u w_{t}^{2} d x \\
& +\frac{\alpha_{13}^{2} a^{2}}{2 \varepsilon b^{2} d_{1}^{2}} \int_{0}^{1} u^{2} v d x+\frac{\varepsilon}{2} \int_{0}^{1} v w_{t}^{2} d x+\frac{\alpha_{13}^{2} A^{2}}{2 \varepsilon B^{2} d_{1}^{2}} \int_{0}^{1} u^{2} w d x+\frac{\varepsilon}{2} \int_{0}^{1} w w_{t}^{2} d x, \\
& \leq \frac{\alpha_{13}^{2}}{2 \varepsilon}\left[1+\left(\frac{a}{b}\right)^{2}+\left(\frac{A}{B}\right)^{2}\right] M_{1}^{2 / 3} d_{1}^{-10 / 3}\left(\int_{0}^{1} u^{5} d x\right)^{1 / 3}+\frac{\alpha_{13}^{2}}{2 \varepsilon} \int_{0}^{1} u^{5} d x \\
& +\varepsilon \int_{0}^{1} u w_{t}^{2} d x+\frac{\varepsilon}{2} \int_{0}^{1} v w_{t}^{2} d x+\frac{\varepsilon}{2} \int_{0}^{1} w w_{t}^{2} d x, \\
& \xi \int_{0}^{1} v_{t} g d x \leq \xi \int_{0}^{1} v_{t} l v\left(d_{1}^{-1}+\frac{e}{b d_{1}}\right) d x \leq \frac{\xi^{2} l^{2} d_{1}^{-2}(1+(e / b))^{2}}{2 \varepsilon} \int_{0}^{1} v d x+\frac{\varepsilon}{2} \int_{0}^{1} v v_{t}^{2} d x \\
& \leq \frac{\xi^{2} l^{2}(1+(e / b))^{2}}{2 \varepsilon} M_{0} d_{1}^{-3}+\frac{\varepsilon}{2} \int_{0}^{1} v v_{t}^{2} d x, \\
& \alpha_{21} \int_{0}^{1} u v_{t} g d x \leq \alpha_{21} \int_{0}^{1} u v_{t} l v\left(d_{1}^{-1}+\frac{e}{b d_{1}}\right) d x \\
& \leq \frac{\alpha_{21}^{2} l^{2} d_{1}^{-2}(1+(e / b))^{2}}{2 \varepsilon} \int_{0}^{1} u^{2} v d x+\frac{\varepsilon}{2} \int_{0}^{1} v v_{t}^{2} d x \\
& \leq \frac{\alpha_{21}^{2} l^{2}(1+(e / b))^{2}}{2 \varepsilon} M_{1}^{2 / 3} d_{1}^{-10 / 3}\left(\int_{0}^{1} u^{5} d x\right)^{1 / 3}+\frac{\varepsilon}{2} \int_{0}^{1} v v_{t}^{2} d x,
\end{aligned}
$$




$$
\begin{aligned}
2 \alpha_{22} \int_{0}^{1} v v_{t} g d x & \leq 2 \alpha_{22} \int_{0}^{1} v^{2} v_{t} l\left(d_{1}^{-1}+\frac{e}{b d_{1}}\right) d x \\
& \leq \frac{\alpha_{22}^{2} l^{2} d_{1}^{-2}(1+(e / b))^{2}}{\varepsilon} \int_{0}^{1} v^{3} d x+\varepsilon \int_{0}^{1} v v_{t}^{2} d x \\
& \leq \frac{\alpha_{21}^{2} l^{2}(1+(e / b))^{2}}{\varepsilon} M_{1}^{2 / 3} d_{1}^{-10 / 3}\left(\int_{0}^{1} v^{5} d x\right)^{1 / 3}+\varepsilon \int_{0}^{1} v v_{t}^{2} d x, \\
\alpha_{23} \int_{0}^{1} w v_{t} g d x & \leq \alpha_{23} \int_{0}^{1} w v_{t} l v\left(d_{1}^{-1}+\frac{e}{b d_{1}}\right) d x \\
& \leq \frac{\alpha_{23}^{2} l^{2} d_{1}^{-2}(1+(e / b))^{2}}{2 \varepsilon} \int_{0}^{1} v^{2} w d x+\frac{\varepsilon}{2} \int_{0}^{1} w v_{t}^{2} d x \\
& \leq \frac{\alpha_{23}^{2} l^{2}(1+(e / b))^{2}}{2 \varepsilon} M_{1}^{2 / 3} d_{1}^{-10 / 3}\left(\int_{0}^{1} v^{5} d x\right)^{1 / 3}+\frac{\varepsilon}{2} \int_{0}^{1} w v_{t}^{2} d x, \\
\alpha_{23}^{1} v w_{t} g d x & \leq \alpha_{23} \int_{0}^{1} v_{t}^{2} w_{t} l\left(d_{1}^{-1}+\frac{e}{b d_{1}}\right) d x \leq \eta \int_{0}^{1} w_{t} L w\left(d_{1}^{-1}+\frac{E}{B d_{1}}\right) d x \\
& \leq \frac{\alpha_{23}^{2} l^{2} d_{1}^{-2}(1+(e / b))^{2}}{2 \varepsilon} \int_{0}^{1} v^{3} d x+\frac{\varepsilon}{2} \int_{0}^{1} v w_{t}^{2} d x \\
& \leq \frac{\alpha_{23}^{2} l^{2}(1+(e / b))^{2}}{2 \varepsilon} M_{1}^{2 / 3} d_{1}^{-10 / 3}\left(\int_{0}^{1} v^{5} d x\right)^{1 / 3}+\frac{\varepsilon}{2} \int_{0}^{1} v w_{t}^{2} d x, \\
\alpha_{21} \int_{0}^{1} v u_{t} g d x & \leq \alpha_{21} \int_{0}^{1} v^{2} v_{t} l\left(d_{1}^{-1}+\frac{e}{b d_{1}}\right) d x \\
& \leq \frac{\alpha_{21}^{2}{ }^{2} d_{1}^{-2}(1+(e / b))^{2}}{2 \varepsilon} \int_{0}^{1} v^{3} d x+\frac{\varepsilon}{2} \int_{0}^{1} v u_{t}^{2} d x \\
& \frac{\alpha_{21}^{2} l^{2}(1+(e / b))^{2}}{2 \varepsilon} M_{1}^{2 / 3} d_{1}^{-10 / 3}\left(\int_{0}^{1} v^{5} d x\right)^{1 / 3}+\frac{\varepsilon}{2} \int_{0}^{1} v u_{t}^{2} d x, \\
&
\end{aligned}
$$


18

Abstract and Applied Analysis

$$
\begin{aligned}
& \leq \frac{\eta^{2} L^{2}(1+(E / B))^{2}}{2 \varepsilon} M_{0} d_{1}^{-3}+\frac{\varepsilon}{2} \int_{0}^{1} w w_{t}^{2} d x, \\
& \alpha_{31} \int_{0}^{1} u w_{t} h d x \leq \alpha_{31} \int_{0}^{1} u w_{t} L w\left(d_{1}^{-1}+\frac{E}{B d_{1}}\right) d x \\
& \leq \frac{\alpha_{31}^{2} L^{2} d_{1}^{-2}(1+(E / B))^{2}}{2 \varepsilon} \int_{0}^{1} w^{2} u d x+\frac{\varepsilon}{2} \int_{0}^{1} u w_{t}^{2} d x \\
& \leq \frac{\alpha_{31}^{2} L^{2}(1+(E / B))^{2}}{2 \varepsilon} M_{1}^{2 / 3} d_{1}^{-10 / 3}\left(\int_{0}^{1} w^{5} d x\right)^{1 / 3}+\frac{\varepsilon}{2} \int_{0}^{1} u w_{t}^{2} d x \\
& \alpha_{32} \int_{0}^{1} v w_{t} h d x \leq \alpha_{32} \int_{0}^{1} v w_{t} L w\left(d_{1}^{-1}+\frac{E}{B d_{1}}\right) d x \\
& \leq \frac{\alpha_{32}^{2} L^{2} d_{1}^{-2}(1+(E / B))^{2}}{2 \varepsilon} \int_{0}^{1} w^{2} v d x+\frac{\varepsilon}{2} \int_{0}^{1} v w_{t}^{2} d x \\
& \leq \frac{\alpha_{32}^{2} L^{2}(1+(E / B))^{2}}{2 \varepsilon} M_{1}^{2 / 3} d_{1}^{-10 / 3}\left(\int_{0}^{1} w^{5} d x\right)^{1 / 3}+\frac{\varepsilon}{2} \int_{0}^{1} v w_{t}^{2} d x \\
& 2 \alpha_{33} \int_{0}^{1} w w_{t} h d x \leq 2 \alpha_{33} \int_{0}^{1} w^{2} w_{t} L\left(d_{1}^{-1}+\frac{E}{B d_{1}}\right) d x \\
& \leq \frac{\alpha_{33}^{2} L^{2} d_{1}^{-2}(1+(E / B))^{2}}{\varepsilon} \int_{0}^{1} w^{3} d x+\varepsilon \int_{0}^{1} w w_{t}^{2} d x \\
& \leq \frac{\alpha_{33}^{2} L^{2}(1+(E / B))^{2}}{\varepsilon} M_{1}^{2 / 3} d_{1}^{-10 / 3}\left(\int_{0}^{1} w^{5} d x\right)^{1 / 3}+\varepsilon \int_{0}^{1} w w_{t}^{2} d x, \\
& \alpha_{31} \int_{0}^{1} w u_{t} h d x \leq \alpha_{31} \int_{0}^{1} w^{2} u_{t} L\left(d_{1}^{-1}+\frac{E}{B d_{1}}\right) d x \\
& \leq \frac{\alpha_{31}^{2} L^{2} d_{1}^{-2}(1+(E / B))^{2}}{2 \varepsilon} \int_{0}^{1} w^{3} d x+\frac{\varepsilon}{2} \int_{0}^{1} w u_{t}^{2} d x \\
& \leq \frac{\alpha_{31}^{2} L^{2}(1+(E / B))^{2}}{2 \varepsilon} M_{1}^{2 / 3} d_{1}^{-10 / 3}\left(\int_{0}^{1} v^{5} d x\right)^{1 / 3}+\frac{\varepsilon}{2} \int_{0}^{1} w u_{t}^{2} d x,
\end{aligned}
$$




$$
\begin{aligned}
\alpha_{32} \int_{0}^{1} w v_{t} h d x & \leq \alpha_{32} \int_{0}^{1} w^{2} v_{t} L\left(d_{1}^{-1}+\frac{E}{B d_{1}}\right) d x \\
& \leq \frac{\alpha_{32}^{2} L^{2} d_{1}^{-2}(1+(E / B))^{2}}{2 \varepsilon} \int_{0}^{1} w^{3} d x+\frac{\varepsilon}{2} \int_{0}^{1} w v_{t}^{2} d x \\
& \leq \frac{\alpha_{32}^{2} L^{2}(1+(E / B))^{2}}{2 \varepsilon} M_{1}^{2 / 3} d_{1}^{-10 / 3}\left(\int_{0}^{1} w^{5} d x\right)^{1 / 3}+\frac{\varepsilon}{2} \int_{0}^{1} w v_{t}^{2} d x
\end{aligned}
$$

By the above inequalities and the condition (1.17), we have

$$
\begin{aligned}
\int_{0}^{1}[(1 & \left.\left.+2 \alpha_{11} u+\alpha_{12} v+\alpha_{13} w\right) u_{t} f+\alpha_{12} u v_{t} f+\alpha_{13} u w_{t} f\right] d x \\
& +\int_{0}^{1}\left[\left(\xi+\alpha_{21} u+2 \alpha_{22} v+\alpha_{23} w\right) v_{t} g+\alpha_{21} v u_{t} g+\alpha_{23} v w_{t} g\right] d x \\
& +\int_{0}^{1}\left[\left(\eta+\alpha_{31} u+\alpha_{32} v+2 \alpha_{33} w\right) w_{t} h+\alpha_{31} w u_{t} h+\alpha_{32} w v_{t} h\right] d x \\
\leq & \bar{\lambda} \varepsilon \int_{0}^{1}(u+v+w)\left(u_{t}^{2}+v_{t}^{2}+w_{t}^{2}\right) d x+\frac{C_{4}}{\varepsilon}\left(1+\xi^{2}+\eta^{2}\right) M_{0} d_{1}^{-3} \\
& +\frac{C_{5}}{\varepsilon}\left(1+d_{1}^{-2}\right) M_{1}^{2 / 3} d_{1}^{-4 / 3}\left[\int_{0}^{1}\left(u^{5}+v^{5}+w^{5}\right) d x\right]^{1 / 3}+\frac{C_{6}}{\varepsilon} \int_{0}^{1}\left(u^{5}+v^{5}+w^{5}\right) d x,
\end{aligned}
$$

where $\bar{\lambda}$ is a constant depending only on $\varepsilon\left(\alpha_{i j}\right)(i, j=1,2,3)$. Choose a small enough positive number $\varepsilon$ which depends on $\alpha_{i j}(i, j=1,2,3), a, b, e, l, A, B, D, E$, and $L$, such that $\bar{\lambda} \varepsilon<C_{3}$. Substituting inequalities (2.24) and (2.26) into (2.21), one can obtain

$$
\begin{aligned}
\frac{1}{2} \bar{y}^{\prime}(t) \leq & -\frac{1}{2} \int_{0}^{1} P_{x x}^{2} d x-\frac{\xi}{2} \int_{0}^{1} Q_{x x}^{2} d x-\frac{\eta}{2} \int_{0}^{1} R_{x x}^{2} d x \\
& +B_{1} K_{2} d_{1}^{-3}+B_{2} Y+B_{3} K_{3} d_{1}^{-2 / 3} Y^{2 / 3}+B_{4} K_{4} d_{1}^{-4 / 3} Y^{1 / 3}
\end{aligned}
$$

where $Y=\int_{0}^{1}\left(u^{5}+v^{5}+w^{5}\right) d x, K_{2}=\left(1+\xi^{2}+\eta^{2}\right) M_{0}+(1+\xi+\eta) M_{1} d_{1}^{-1}, K_{3}=M_{1}^{1 / 3}, K_{4}=$ $M_{1}^{2 / 3}\left(1+d_{1}^{-2}\right)$.

Note that

$$
P \geq \alpha_{11} u^{2}, \quad Q \geq \alpha_{22} v^{2}, \quad R \geq \alpha_{33} w^{2} .
$$


It follows from (2.18) and (2.4) to functions $P, Q, R$ that

$$
\begin{aligned}
& Y \leq B_{5} \int_{0}^{1}\left(P^{5 / 2}+Q^{5 / 2}+R^{5 / 2}\right) d x \leq B_{6} K_{1}^{3 / 2} d_{1}^{-3 / 2} \bar{y}^{1 / 2}+B_{6} K_{1}^{5 / 2} d_{1}^{-5 / 2}, \\
& Y^{1 / 3} \leq B_{7} K_{1}^{1 / 2} d_{1}^{-1 / 2} \bar{y}^{1 / 6}+B_{7} K_{1}^{5 / 6} d_{1}^{-5 / 6} \\
& Y^{2 / 3} \leq B_{8} K_{1} d_{1}^{-1} \bar{y}^{1 / 3}+B_{8} K_{1}^{5 / 3} d_{1}^{-5 / 3}
\end{aligned}
$$

Moreover, one can obtain by (2.5) and (2.18) that

$$
\begin{aligned}
& -\frac{1}{2} \int_{0}^{1} P_{x x}^{2} d x-\frac{\xi}{2} \int_{0}^{1} Q_{x x}^{2} d x-\frac{\eta}{2} \int_{0}^{1} R_{x x}^{2} d x \\
& \quad \leq-B_{9} \min \{1, \xi, \eta\} K_{1}^{-4 / 3} d_{1}^{4 / 3} \bar{y}^{5 / 3}+(1+\xi+\eta) K_{1}^{2} d_{1}^{-2} .
\end{aligned}
$$

Combining (2.27),(2.29), and (2.30), we have

$$
\begin{aligned}
\frac{1}{2} \bar{y}^{\prime}(t) \leq & -A_{1} \min \{1, \xi, \eta\} K_{1}^{-4 / 3} d_{1}^{4 / 3} \bar{y}^{5 / 3} \\
& +A_{2}\left[(1+\xi+\eta) K_{1}^{2} d_{1}^{-2}+K_{2} d_{1}^{-3}+K_{1}^{5 / 2} d_{1}^{-5 / 2}+K_{1}^{5 / 3} K_{3} d_{1}^{-7 / 3}+K_{1}^{5 / 6} K_{4} d_{1}^{-13 / 6}\right] \\
& +A_{3} K_{1}^{3 / 2} d_{1}^{-3 / 2} \bar{y}^{1 / 2}+A_{4} K_{1} K_{3} d_{1}^{-5 / 3} \bar{y}^{1 / 3}+A_{5} K_{1}^{1 / 2} K_{4} d_{1}^{-11 / 6} \bar{y}^{1 / 6}
\end{aligned}
$$

Multiplying inequality (2.31) by $d_{1}^{2}$, we have

$$
\begin{aligned}
\frac{1}{2} y^{\prime}(t) \leq & -A_{1} \min \{1, \xi, \eta\} K_{1}^{-4 / 3} y^{5 / 3} \\
& +A_{2}\left[(1+\xi+\eta) K_{1}^{2}+K_{2} d_{1}^{-1}+K_{1}^{5 / 2} d_{1}^{-1 / 2}+K_{1}^{5 / 3} K_{3} d_{1}^{-1 / 3}+K_{1}^{5 / 6} K_{4} d_{1}^{-1 / 6}\right] \\
& +A_{3} K_{1}^{3 / 2} d_{1}^{-1 / 2} y^{1 / 2}+A_{4} K_{1} K_{3} d_{1}^{-1 / 3} y^{1 / 3}+A_{5} K_{1}^{1 / 2} K_{4} d_{1}^{-1 / 6} y^{1 / 6}
\end{aligned}
$$

where $y=\int_{0}^{1}\left[\left(d_{1} P_{x}\right)^{2}+\left(d_{1} Q_{x}\right)^{2}+\left(d_{1} R_{x}\right)^{2}\right] d x$. The inequality (2.32) implies that there exist $\widetilde{\tau}_{2}>0$ and positive constant $\widetilde{M}_{2}$ depending on $d_{i}, \alpha_{i j}(i, j=1,2,3), a, b, e, l, A, B, D, E$, and $L$, such that

$$
\int_{0}^{1}\left(d_{1} P_{x}\right)^{2} d x, \int_{0}^{1}\left(d_{1} Q_{x}\right)^{2} d x, \int_{0}^{1}\left(d_{1} R_{x}\right)^{2} d x \leq \widetilde{M}_{2}, \quad t \geq \widetilde{\tau}_{2}
$$

In the case that $d_{1}, d_{2}, d_{3} \geq 1, \xi, \eta \in[\underline{d}, \bar{d}]$, the coefficients of inequality (2.31) can be estimated by some constants which depend on $\underline{d}, \bar{d}$, but do not depend on $d_{1}, d_{2}, d_{3}$. So $\widetilde{M}_{2}$ depends 
on $\alpha_{i j}(i, j=1,2,3), a, b, e, l, A, B, D, E, L, \underline{d}$, and $\bar{d}$, but it is irrelevant to $d_{1}, d_{2}, d_{3}$, when $d_{1}, d_{2}, d_{3} \geq 1$ and $\xi, \eta \in[\underline{d}, \bar{d}]$. Since

$$
\left(\begin{array}{c}
P_{x} \\
Q_{x} \\
R_{x}
\end{array}\right)=\left(\begin{array}{lll}
P_{u} & P_{v} & P_{w} \\
Q_{u} & Q_{v} & Q_{w} \\
R_{u} & R_{v} & R_{w}
\end{array}\right)\left(\begin{array}{c}
u_{x} \\
v_{x} \\
w_{x}
\end{array}\right)
$$

we can transform the formulations of $u_{x}, v_{x}, w_{x}$ into fraction representations, then distribute the denominators of the absolute value of the fractions to the numerators item and enlarge the term concerning with $u_{x}, v_{x}$ or $w_{x}$ to obtain

$$
\left|d_{1} u_{x}\right|+\left|d_{1} v_{x}\right|+\left|d_{1} w_{x}\right| \leq C^{\prime}\left(\left|d_{1} P_{x}\right|+\left|d_{1} Q_{x}\right|+\left|d_{1} R_{x}\right|\right), \quad 0<x<1, t>0,
$$

where $C^{\prime}$ is a constant depending only on $\xi, \eta, \alpha_{i j}(i, j=1,2,3)$. After scaling back and contacting estimates (2.33) and (2.35), there exist positive constant $M_{2}$ depending on $d_{i}, \alpha_{i j}(i, j=1,2,3), a, b, e, l, A, B, D, E, L$, and $\tau_{2}>0$, such that

$$
\int_{0}^{1} u_{x}^{2} d x, \int_{0}^{1} v_{x}^{2} d x, \int_{0}^{1} w_{x}^{2} d x \leq M_{2}, \quad t \geq \tau_{2}
$$

When $d_{1}, d_{2}, d_{3} \geq 1$ and $\xi, \eta \in[\underline{d}, \bar{d}], M_{2}$ is independent of $d_{1}, d_{2}, d_{3}$.

(ii) One has $t \geq 0$. Modifying the dependency of the coefficients in inequalities (2.17)(2.22), namely, replacing $M_{0}, M_{1}$ with $M_{0}^{\prime}, M_{1}^{\prime}$, there exists a positive constant $M_{2}^{\prime}$ depending on $d_{i}, \alpha_{i j}(i, j=1,2,3), a, b, e, l, A, B, \mathrm{D}, E, L$, and the $W_{2}^{1}$-norm of $u_{0}, v_{0}, w_{0}$, such that

$$
\int_{0}^{1} u_{x}^{2} d x, \int_{0}^{1} v_{x}^{2} d x, \int_{0}^{1} w_{x}^{2} d x \leq M_{2}^{\prime}, \quad t \geq 0
$$

Furthermore, in the case that $d_{1}, d_{2}, d_{3} \geq 1, \xi, \eta \in[\underline{d}, \bar{d}], M_{2}^{\prime}$ depends on $\underline{d}, \bar{d}$, but does not depend on $d_{1}, d_{2}, d_{3}$.

Summarizing estimates (2.8), (2.15), (2.36) and Sobolev embedding theorem, there exist positive constants $M, M^{\prime}$ depending only on $d_{i}, \alpha_{i j}(i, j=1,2,3), a, b, e, l, A, B, D, E$, and $L$, such that (1.18) and (1.19) hold. In particular, $M, M^{\prime}$ depend only on $\alpha_{i j}(i, j=$ $1,2,3), a, b, e, l, A, B, D, E, L, \underline{d}$, and $\bar{d}$, but do not depend on $d_{1}, d_{2}, d_{3}$, when $d_{1}, d_{2}, d_{3} \geq 1$ and $\xi, \eta \in[\underline{d}, \bar{d}]$.

Similarly, according to $(2.8)^{\prime},(2.15)^{\prime},(2.36)^{\prime}$, there exists a positive constant $M^{\prime \prime}$ depending on $d_{i}, \alpha_{i j}(i, j=1,2,3), a, b, e, l, A, B, D, E, L$ and the initial functions $u_{0}, v_{0}, w_{0}$, such that

$$
|u(\cdot, t)|_{1,2},|v(\cdot, t)|_{1,2},|w(\cdot, t)|_{1,2} \leq M^{\prime \prime}, \quad t \geq 0 .
$$

Further, in the case that $d_{1}, d_{2}, d_{3} \geq 1, \xi, \eta \in[\underline{d}, \bar{d}], M^{\prime \prime}$ depends only on $\underline{d}, \bar{d}$, but do not depend on $d_{1}, d_{2}, d_{3}$. Thus, $T=+\infty$. This completes the proof of Theorem 1.1. 


\section{Global Stability}

In order to obtain the uniform convergence of the solution for the system (1.16), we recall the following result which can be found in [37, 42].

Lemma 3.1. Let $a$ and $b$ be positive constants. Assume that $\varphi, \psi \in C^{1}([a,+\infty)), \psi(t) \geq 0$ and $\varphi$ is bounded from below. If $\varphi^{\prime}(t) \leq-b \psi(t)$ and $\psi^{\prime}(t)$ is bounded from above in $[a,+\infty)$, then $\lim _{t \rightarrow \infty} \psi(t)=0$.

Proof of Theorem 1.3. Let $(u, v, w)$ be a solution for the system (1.16) with initial functions $u_{0}(x), v_{0}(x), w_{0}(x) \geq(\not \equiv) 0$. From the strong maximum principle for parabolic equations, it is not hard to verify that $u, v, w>0$ for $t>0$. Define the function

$$
\begin{aligned}
H(u, v, w)= & \int_{0}^{1}\left(u-u^{*}-u^{*} \ln \frac{u}{u^{*}}\right) d x+\alpha \int_{0}^{1}\left(v-v^{*}-v^{*} \ln \frac{v}{v^{*}}\right) d x \\
& +\beta \int_{0}^{1}\left(w-w^{*}-w^{*} \ln \frac{w}{w^{*}}\right) d x .
\end{aligned}
$$

Then the time derivative of $H(u, v, w)$ for the system (1.16) satisfies

$$
\begin{aligned}
\frac{d H}{d t}= & \int_{0}^{1} \frac{u-u^{*}}{u} u_{t} d x+\alpha \int_{0}^{1} \frac{v-v^{*}}{v} v_{t} d x+\beta \int_{0}^{1} \frac{w-w^{*}}{w} w_{t} d x \\
= & -\int_{0}^{1}\left[\frac{u^{*}}{u^{2}}\left(d_{1}+2 \alpha_{11} u+\alpha_{12} v+\alpha_{13} w\right) u_{x}^{2}+\frac{u^{*}}{u}\left(\alpha_{12} v_{x}+\alpha_{13} w_{x}\right) u_{x}\right. \\
& +\frac{\alpha v^{*}}{v^{2}}\left(d_{2}+\alpha_{21} u+2 \alpha_{22} v+\alpha_{23} w\right) v_{x}^{2}+\frac{\alpha v^{*}}{v}\left(\alpha_{21} u_{x}+\alpha_{23} w_{x}\right) v_{x} \\
& \left.+\frac{\beta w^{*}}{w^{2}}\left(d_{3}+\alpha_{31} u+\alpha_{32} v+2 \alpha_{33} w\right) w_{x}^{2}+\frac{\beta w^{*}}{w}\left(\alpha_{31} u_{x}+\alpha_{13} v_{x}\right) w_{x}\right] d x \\
& +\int_{0}^{1}\left(u-u^{*}\right)\left(1-u-\frac{a v}{1+b u}-\frac{A w}{1+B u+D w}\right) d x+\alpha \int_{0}^{1}\left(v-v^{*}\right) m\left(-1+\frac{e u}{1+b u}\right) d x \\
& +\beta \int_{0}^{1}\left(w-w^{*}\right) M\left(-1+\frac{E u}{1+B u+D w}\right) d x
\end{aligned}
$$

The first integrand in the right hand of (3.2) is positive definite if

$$
\begin{aligned}
4 \alpha \beta u^{*} v^{*} w^{*}\left(d_{1}+2 \alpha_{11} u+\alpha_{12} v+\alpha_{13} w\right)\left(d_{2}+\alpha_{21} u+2 \alpha_{22} v+\alpha_{23} w\right)\left(d_{3}+\alpha_{31} u+\alpha_{32} v+2 \alpha_{33} w\right) \\
>u^{*}\left(\alpha \alpha_{23} v^{*} w+\beta \alpha_{32} w^{*} v\right)^{2}\left(d_{1}+2 \alpha_{11} u+\alpha_{12} v+\alpha_{13} w\right) \\
\quad+\alpha v^{*}\left(\alpha_{13} u^{*} w+\beta \alpha_{31} w^{*} u\right)^{2}\left(d_{2}+\alpha_{21} u+2 \alpha_{22} v+\alpha_{23} w\right) \\
+\beta w^{*}\left(\alpha_{12} u^{*} v+\alpha \alpha_{21} v^{*} u\right)^{2}\left(d_{3}+\alpha_{31} u+\alpha_{32} v+2 \alpha_{33} w\right) .
\end{aligned}
$$


By the maximum-norm estimate in Theorem 1.1, the condition (1.20) implies (3.3). Therefore, we have

$$
\begin{aligned}
\frac{d H}{d t} \leq & -\int_{0}^{1}\left[1-\frac{a b v^{*}}{\left(1+b u^{*}\right)(1+b u)}-\frac{A B w^{*}}{\left(1+B u^{*}+D w^{*}\right)(1+B u+D w)}\right]\left(u-u^{*}\right)^{2} d x \\
& -\beta \int_{0}^{1} \frac{M D E u^{*}}{\left(1+B u^{*}+D w^{*}\right)(1+B u+D w)}\left(w-w^{*}\right)^{2} d x \\
\leq & -\int_{0}^{1}\left[1-\frac{a b v^{*}}{1+b u^{*}}-\frac{A B w^{*}}{1+B u^{*}+D w^{*}}\right]\left(u-u^{*}\right)^{2} d x-\beta \int_{0}^{1} \frac{M D E u^{*}}{1+B u^{*}+D w^{*}}\left(w-w^{*}\right)^{2} d x \\
\triangleq & -l_{1} \int_{0}^{1}\left(u-u^{*}\right)^{2} d x-\beta l_{2} \int_{0}^{1}\left(w-w^{*}\right)^{2} d x
\end{aligned}
$$

where

$$
\begin{gathered}
l_{1}=1-\frac{a b v^{*}}{1+b u^{*}}-\frac{A B w^{*}}{1+B u^{*}+D w^{*}} \\
l_{2}=\frac{M D E u^{*}}{1+B u^{*}+D w^{*}}>0 .
\end{gathered}
$$

The condition (1.21) implies $l_{1}>0$. Using the similar argument in the proof of Theorem 4.2 in [42], by the maximum-norm estimate in Theorem 1.1 and some tedious calculations, we can prove

$$
\lim _{t \rightarrow \infty} \int_{0}^{1}\left(u-u^{*}\right)^{2} d x=\lim _{t \rightarrow \infty} \int_{0}^{1}\left(v-v^{*}\right)^{2} d x=\lim _{t \rightarrow \infty} \int_{0}^{1}\left(w-w^{*}\right)^{2} d x=0
$$

It follows from (3.6) and Gagliardo-Nirenberg-type inequality $|u|_{\infty} \leq C|u|_{1,2}^{1 / 2}|u|_{2}^{1 / 2}$ that $(u, v, w)$ converges uniformly on $\left(u^{*}, v^{*}, w^{*}\right)$. By the fact that $H(u, v, w)$ is decreasing for $t \geq$ 0 , it is obvious that $\left(u^{*}, v^{*}, w^{*}\right)$ is globally asymptotically stable. So the proof of Theorem 1.3 is completed.

\section{Acknowledgments}

This work is supported by the China National Natural Science Foundation (no. 11061031; 11161041), the Fundamental Research Funds for the Gansu University, NWNU-KJCXGC-0361 and the Fundamental Research Funds for the Central Universities (no. zyz2012074).

\section{References}

[1] V. Volterra, "Variations and fluctuations of the number of individuals in animal species living together," Journal du Conseil Conseil International pour l'Exploration de la Mer, vol. 3, pp. 3-51, 1928.

[2] F. J. Ayala, "Experimental invalidation of the principle of competitive exclusion," Nature, vol. 224, pp. 1076-1079, 1969. 
[3] R. S. Cantrell and C. Cosner, Spatial Ecology via Reaction-Diffusion Equations, Wiley Series in Mathematical and Computational Biology, John Wiley \& Sons, Chichester, UK, 2003.

[4] T. W. Schoener, "Alternatives to Lotka-Volterra competition: models of intermediate complexity," Theoretical Population Biology, vol. 10, no. 3, pp. 309-333, 1976.

[5] R. S. Cantrell, C. Cosner, and S. Ruan, "Intraspecific interference and consumer-resource dynamics," Discrete and Continuous Dynamical Systems Series B, vol. 4, no. 3, pp. 527-546, 2004.

[6] L.-J. Hei and Y. Yu, "Non-constant positive steady state of one resource and two consumers model with diffusion," Journal of Mathematical Analysis and Applications, vol. 339, no. 1, pp. 566-581, 2008.

[7] K. Kuto, "Stability of steady-state solutions to a prey-predator system with cross-diffusion," Journal of Differential Equations, vol. 197, no. 2, pp. 293-314, 2004.

[8] K. Kuto and Y. Yamada, "Multiple coexistence states for a prey-predator system with cross-diffusion," Journal of Differential Equations, vol. 197, no. 2, pp. 315-348, 2004.

[9] Y. Lou and W. Ni, "Diffusion, self-diffusion and cross-diffusion," Journal of Differential Equations, vol. 131, pp. 79-131, 1996.

[10] Y. Lou and W.-M. Ni, "Diffusion vs cross-diffusion: an elliptic approach," Journal of Differential Equations, vol. 154, no. 1, pp. 157-190, 1999.

[11] P. Y. H. Pang and M. Wang, "Strategy and stationary pattern in a three-species predator-prey model," Journal of Differential Equations, vol. 200, no. 2, pp. 245-273, 2004.

[12] J. Shi, Z. Xie, and K. Little, "Cross-diffusion induced instability and stability in reaction-diffusion systems," Journal of Applied Analysis and Computation, vol. 1, pp. 95-119, 2011.

[13] C. Tian, Z. Lin, and M. Pedersen, "Instability induced by cross-diffusion in reaction-diffusion systems," Nonlinear Analysis. Real World Applications, vol. 11, no. 2, pp. 1036-1045, 2010.

[14] M. Wang, "Stationary patterns of strongly coupled prey-predator models," Journal of Mathematical Analysis and Applications, vol. 292, no. 2, pp. 484-505, 2004.

[15] Y. S. Choi, R. Lui, and Y. Yamada, "Existence of global solutions for the Shigesada-Kawasaki-Teramoto model with weak cross-diffusion," Discrete and Continuous Dynamical Systems Series A, vol. 9, no. 5, pp. 1193-1200, 2003.

[16] Y.S. Choi, R. Lui, and Y. Yamada, "Existence of global solutions for the Shigesada-Kawasaki-Teramoto model with strongly coupled cross-diffusion," Discrete and Continuous Dynamical Systems Series A, vol. 10, no. 3, pp. 719-730, 2004.

[17] S. Fu, Z. Wen, and S. Cui, "Uniform boundedness and stability of global solutions in a strongly coupled three-species cooperating model," Nonlinear Analysis: Real World Applications, vol. 55, pp. $1-18,2006$.

[18] D. Le, "Cross diffusion systems on $n$ spatial dimensional domains," Indiana University Mathematics Journal, vol. 51, no. 3, pp. 625-643, 2002.

[19] D. Le, L. V. Nguyen, and T. T. Nguyen, "Shigesada-Kawasaki-Teramoto model on higher dimensional domains," Electronic Journal of Differential Equations, vol. 72, pp. 1-12, 2003.

[20] Y. Lou, W.-M. Ni, and Y. Wu, "On the global existence of a cross-diffusion system," Discrete and Continuous Dynamical Systems, vol. 4, no. 2, pp. 193-203, 1998.

[21] P. V. Tuoc, "Global existence of solutions to Shigesada-Kawasaki-Teramoto cross-diffusion systems on domains of arbitrary dimensions," Proceedings of the American Mathematical Society, vol. 135, no. 12, pp. 3933-3941, 2007.

[22] P. V. Tuoc, "On global existence of solutions to a cross-diffusion system," Journal of Mathematical Analysis and Applications, vol. 343, no. 2, pp. 826-834, 2008.

[23] Y. Yamada, "Existence of global solutions for the Shigesada-Kawasaki-Teramoto model with crossdiffusion," Mathematical and Analytic Institute Communications in Waseda University, vol. 1358, pp. 24 33, 2004.

[24] J. W. Barrett and J. F. Blowey, "Finite element approximation of a nonlinear cross-diffusion population model," Numerische Mathematik, vol. 98, no. 2, pp. 195-221, 2004.

[25] J. W. Barrett, J. F. Blowey, and H. Garcke, "Finite element approximation of a fourth order nonlinear degenerate parabolic equation," Numerische Mathematik, vol. 80, no. 4, pp. 525-556, 1998.

[26] J. W. Barrett, H. Garcke, and R. Nürnberg, "Finite element approximation of surfactant spreading on a thin film," SIAM Journal on Numerical Analysis, vol. 41, no. 4, pp. 1427-1464, 2003.

[27] L. Chen and A. Jüngel, "Analysis of a parabolic cross-diffusion population model without selfdiffusion," Journal of Differential Equations, vol. 224, no. 1, pp. 39-59, 2006.

[28] F. Filbet, "A finite volume scheme for the Patlak-Keller-Segel chemotaxis model," Numerische Mathematik, vol. 104, no. 4, pp. 457-488, 2006. 
[29] G. Galiano, M. L. Garzón, and A. Jüngel, "Semi-discretization in time and numerical convergence of solutions of a nonlinear cross-diffusion population model," Numerische Mathematik, vol. 93, no. 4, pp. 655-673, 2003.

[30] G. Gilardi and U. Stefanelli, "Time-discretization and global solution for a doubly nonlinear Volterra equation," Journal of Differential Equations, vol. 228, no. 2, pp. 707-736, 2006.

[31] Y.-H. Fan, L.-L. Wang, and M.-X. Wang, "Notes on multiple bifurcations in a delayed predator-prey model with nonmonotonic functional response," International Journal of Biomathematics, vol. 2, no. 2, pp. 129-138, 2009.

[32] Z. Luo and Z.-R. He, "Optimal harvesting problem for an age-dependent $n$-dimensional competing system with diffusion," International Journal of Biomathematics, vol. 1, no. 2, pp. 133-145, 2008.

[33] B. Dubey and B. Hussain, "A predator-prey interaction model with self and cross-diffusion," Ecological Modelling, vol. 141, pp. 67-76, 2001.

[34] H. Amann, "Dynamic theory of quasilinear parabolic equations-I. Abstract evolution equations," Nonlinear Analysis. Theory, Methods E Applications, vol. 12, no. 9, pp. 895-919, 1988.

[35] H. Amann, "Dynamic theory of quasilinear parabolic equations-II. Reaction-diffusion systems," Differential and Integral Equations, vol. 3, no. 1, pp. 13-75, 1990.

[36] H. Amann, "Dynamic theory of quasilinear parabolic systems-III. Global existence," Mathematische Zeitschrift, vol. 202, no. 2, pp. 219-250, 1989.

[37] M. Wang, Nonlinear Partial Differential Equations of Parabolic Type, Science Press, Beijing, China, 1993.

[38] S.-A. Shim, "Uniform boundedness and convergence of solutions to cross-diffusion systems," Journal of Differential Equations, vol. 185, no. 1, pp. 281-305, 2002.

[39] S.-A. Shim, "Uniform boundedness and convergence of solutions to the systems with cross-diffusions dominated by self-diffusions," Nonlinear Analysis. Real World Applications, vol. 4, no. 1, pp. 65-86, 2003.

[40] S.-A. Shim, "Uniform boundedness and convergence of solutions to the systems with a single nonzero cross-diffusion," Journal of Mathematical Analysis and Applications, vol. 279, no. 1, pp. 1-21, 2003.

[41] L. Nirenberg, "On elliptic partial differential equations," Annali della Scuola Normale Superiore di Pisa, vol. 13, pp. 115-162, 1959.

[42] Z. Lin and M. Pedersen, "Stability in a diffusive food-chain model with Michaelis-Menten functional response," Nonlinear Analysis. Theory, Methods \& Applications, vol. 57, no. 3, pp. 421-433, 2004. 


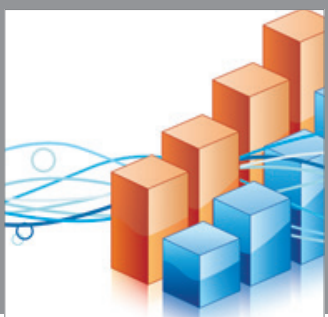

Advances in

Operations Research

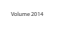

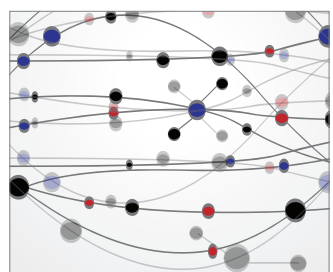

\section{The Scientific} World Journal
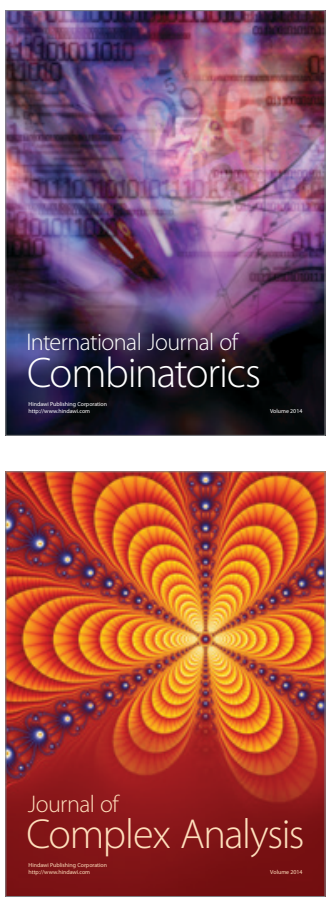

International Journal of

Mathematics and

Mathematical

Sciences
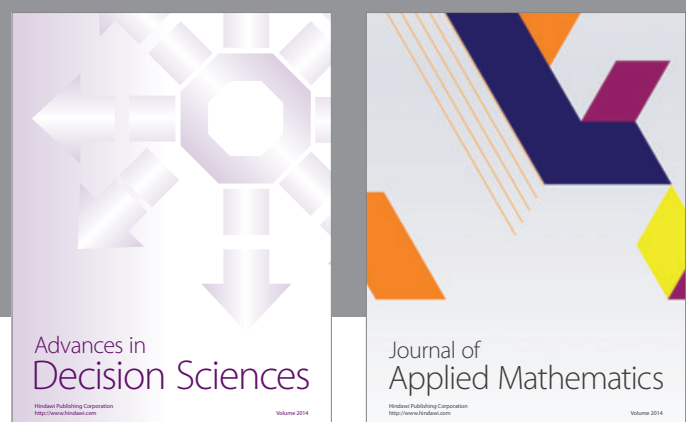

Journal of

Applied Mathematics
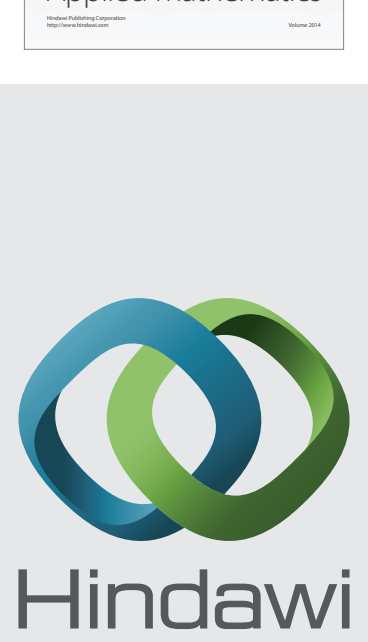

Submit your manuscripts at http://www.hindawi.com
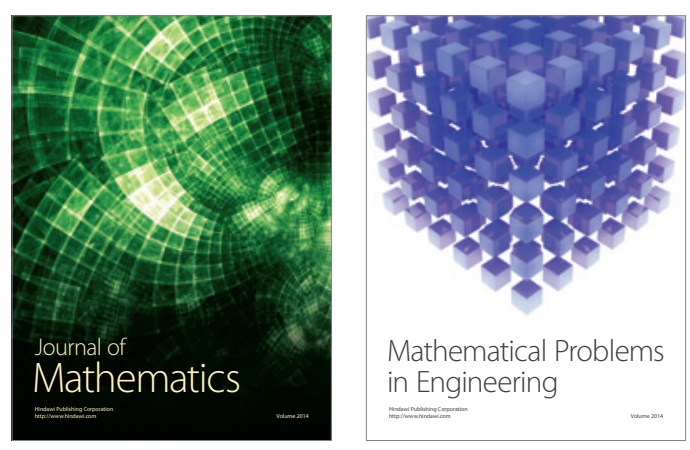

Mathematical Problems in Engineering
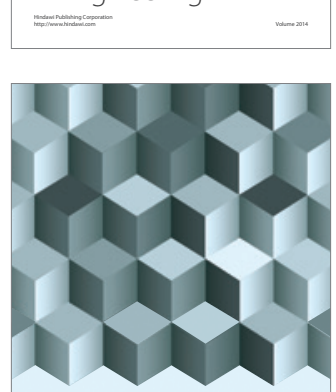

Journal of

Function Spaces
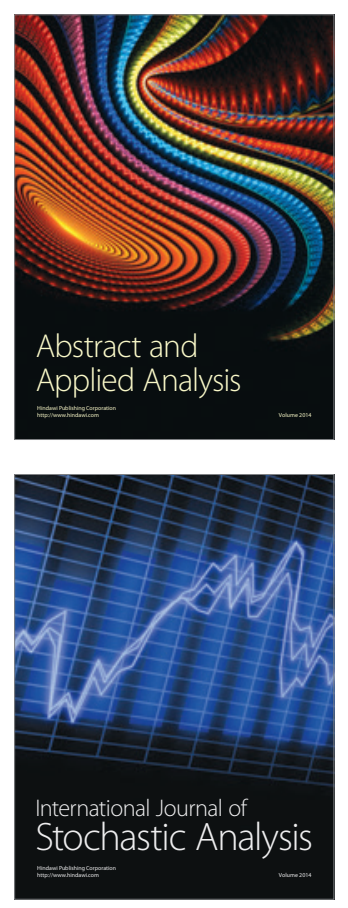

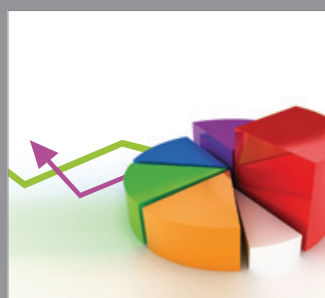

ournal of

Probability and Statistics

Promensencen
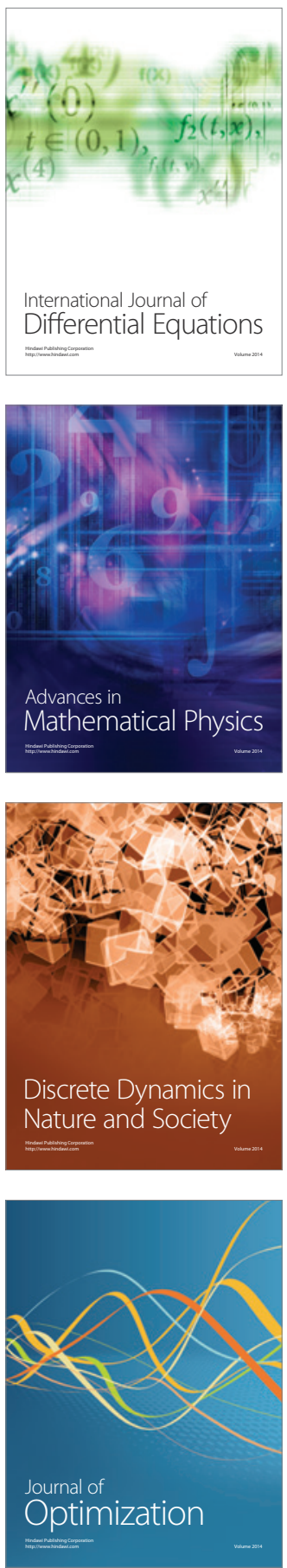